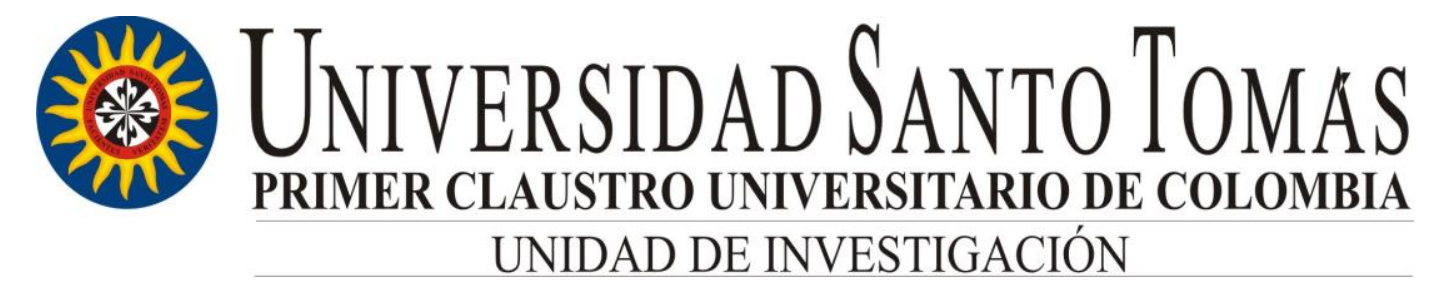

\title{
DEMANDA DE TRANSPORTE EN LA CIUDAD DE MEDELLIN, COLOMBIA. PERIODO 2017
}

Ing. Civil Mauricio Arturo Molina Montenegro

Trabajo de grado para optar el título de Magister en Ciencias Económicas

Ph.D. Econ. Gustavo Adolfo Junca Rodríguez

Facultad de Economía

Maestría en Ciencias Económicas

Bogotá. D.C.

2019 


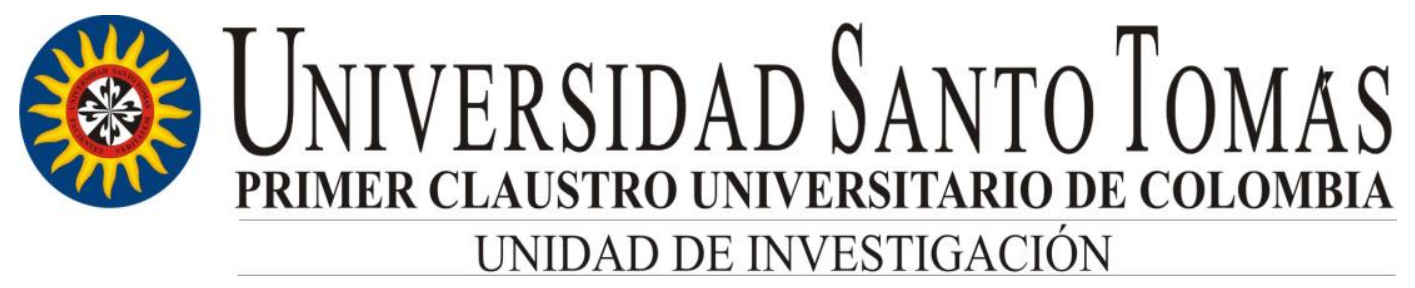

Agradecimientos

Este documento hace parte de la culminación de un proceso de formación personal, quiero dedicarle el título de Magister en Ciencias Económicas a mis hijos Luna Antonia Brisa De Primavera y Emilio Sol Cristóbal, por supuesto también a mis padres Martha Cecilia y Héctor Orlando, dado que no hubiera sido posible obtener este triunfo sin su apoyo total.

No puedo olvidar personas dentro de mis afectos como Olga Lucia, a quien también dedico la culminación de esta etapa como una muestra de gratitud por su apoyo.

A su vez manifiesto mi especial agradecimiento a la planta docente de la Maestría en Ciencias Económicas de mi Alma Mater, y en especial a Andrés Vernazza y Gustavo Junca por su permanente orientación y apoyo. 


\section{Universidad SANTOTOMaS PRIMER CLAUSTRO UNIVERSITARIO DE COLOMBIA UNIDAD DE INVESTIGACIÓN}

\section{Resumen}

El Bienestar Social es la consecuencia de una adecuada política pública, se enmarca en la capacidad de garantizar el acceso de una comunidad a las diferentes oportunidades que se generan en su entorno. La movilidad urbana facilita el acceso a oportunidades que se evidencian con la calidad de vida de las personas, puesto que el transporte público es la forma más económica de desplazamiento dentro de una urbe o área metropolitana.

Las ciudades que implementan servicios de transporte integrado y moderno generan el desarrollo de políticas públicas que conllevan a resultados positivos en términos económicos. Las principales evidencias empíricas demuestran un aumento en el empleo para ciudades que implementan transportes modernos e integrados.

Para lograr beneficios económicos en una ciudad, la accesibilidad juega un papel determinante y por eso es el eje central del Reporte de Economía y Desarrollo (RED) 2017 de CAF -banco de desarrollo de América Latina- titulado "Crecimiento urbano y acceso a oportunidades: un desafío para América Latina".

En el año 2017, el Área Metropolitana del Valle de Aburrá (AMVA), realizo la encuesta Origen-Destino (EOD) a 16.340 hogares en zonas urbanas y rurales del Valle de Aburrá, y con estos datos se presenta una herramienta diagnostica para tomar decisiones frente al desarrollo territorial en materia de movilidad y cuyos datos sirven como eje de la presente investigación.

Palabras Clave: Bienestar Social, calidad de vida, objetivos desarrollo sostenible, disminución de pobreza, generación de empleo, sistemas de transporte, metro, metro cable.

Clasificación JEL: O18, P25, R41, L92 


\title{
Universidad SANTOTOMas PRIMER CLAUSTRO UNIVERSITARIO DE COLOMBIA UNIDAD DE INVESTIGACIÓN
}

\begin{abstract}
Social Welfare is the consequence of an adequate public policy, is framed in the ability to guarantee the access of a community to the different opportunities that are generated in its environment. Urban mobility facilitates access to opportunities that are evidenced by people's quality of life, since public transport is the most economical form of travel within a city or metropolitan area.

The cities that implement integrated and modern transport services generate the development of public policies that lead to positive results in economic terms. The main empirical evidence demonstrates an increase in employment for cities that implement modern and integrated transport.

To achieve economic benefits in a city, accessibility plays a decisive role and that is why it is the central axis of CAF's 2017 Economy and Development Report (RED) - development bank of Latin America - entitled "Urban growth and access to opportunities: a challenge for Latin America. "

In 2017, the Aburrá Valley Metropolitan Area conducted the Origin-Destination survey of 16,340 households in urban and rural areas of the Aburrá Valley, and with these data a diagnostic tool is presented to make decisions regarding territorial development regarding mobility and whose data serve as the axis of the present investigation.
\end{abstract}

Keywords: Social Welfare, quality of life, sustainable development goals, poverty reduction, job creation, transportation systems, subway, cable subway.

JEL Classification: O18, P25, R41, L92 


\section{Universidad SANTOTOMas PRIMER CLAUSTRO UNIVERSITARIO DE COLOMBIA UNIDAD DE INVESTIGACIÓN}

\section{Résumé:}

La protection sociale est la conséquence d'une politique publique adéquate. Elle s'articule autour de la capacité de garantir l'accès d'une communauté aux différentes opportunités générées dans son environnement. La mobilité urbaine facilite l'accès aux opportunités mises en évidence par la qualité de vie des personnes, les transports en commun étant la forme de transport la plus économique dans une ville ou une région métropolitaine.

Les villes qui mettent en œuvre des services de transport intégrés et modernes génèrent l'élaboration de politiques publiques qui produisent des résultats positifs en termes économiques. Les principales preuves empiriques montrent une augmentation de l'emploi dans les villes mettant en œuvre des transports modernes et intégrés.

L'accessibilité joue un rôle décisif dans l'atteinte des retombées économiques dans une ville. C'est pourquoi elle constitue l'axe central du Rapport sur l'économie et le développement (RED) 2017 de la CAF - Banque de développement de l'Amérique latine - intitulé "Croissance urbaine et accès aux opportunités: un défi pour l'Amérique latine. "

En 2017, la région métropolitaine de la vallée d'Aburrá a mené l'enquête Origine-Destination auprès de 16340 ménages des zones urbaines et rurales de la vallée d'Aburrá. À partir de ces données, un outil de diagnostic est présenté pour prendre des décisions en matière de développement territorial en matière de mobilité. axe de la présente enquête.

Mots-clés: protection sociale, qualité de vie, objectifs de développement durable, réduction de la pauvreté, création d'emplois, systèmes de transport, métro, métro à câbles.

Classification JEL: O18, P25, R41, L92 


\section{Contenido}

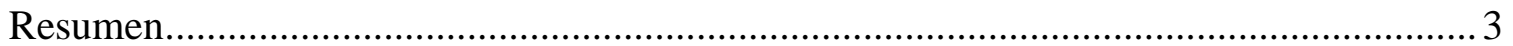

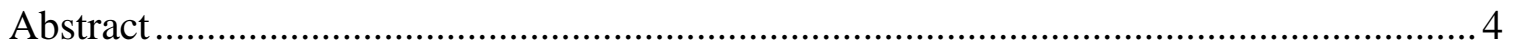

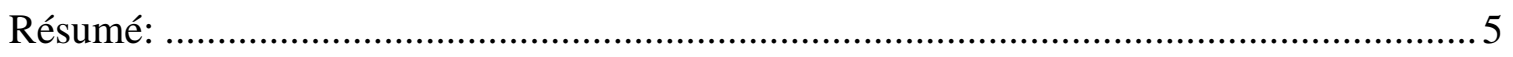

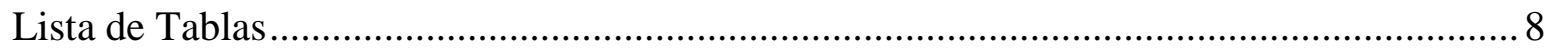

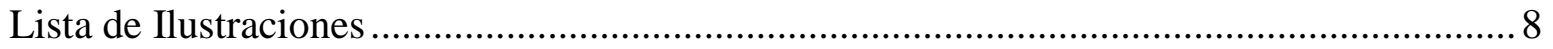

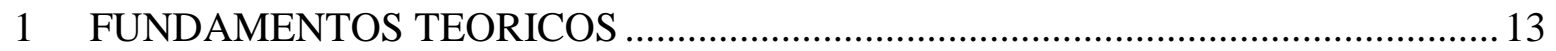

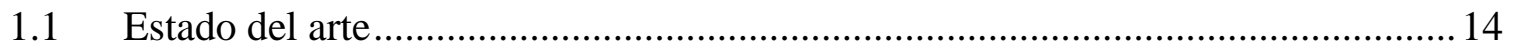

1.2 Estructuras de oportunidades de movilidad ...................................................... 16

1.3 Estudios de transporte en oportunidades económicas......................................... 19

2 CARACTERIZACIÓN DEL TRANSPORTE PUBLICO EN MEDELLIN................20

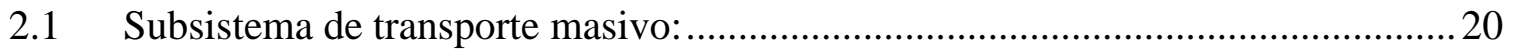

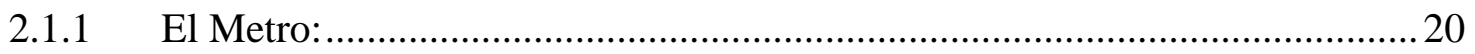

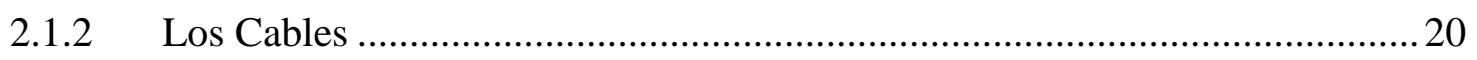

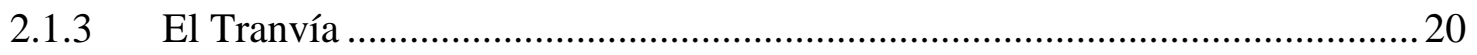

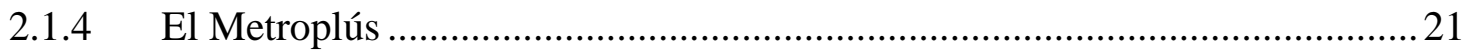

2.1.5 Buses alimentadores y rutas integradas ................................................... 21

2.2 Subsistema de transporte público colectivo de pasajeros (TPC): ........................21

2.3 Sistema de bicicletas públicas del Valle de Aburrá - Encicla..............................21

3 METODOLOGIA Y FUENTES DE INFORMACIÓN .......................................23

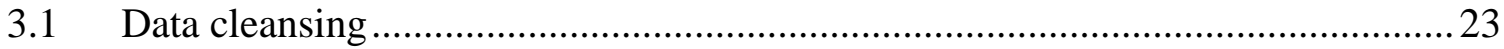

3.2 Consideraciones éticas y recolección de datos ................................................23

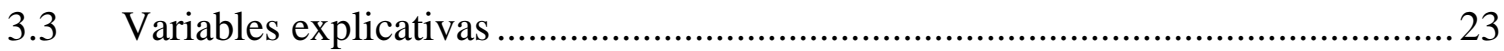

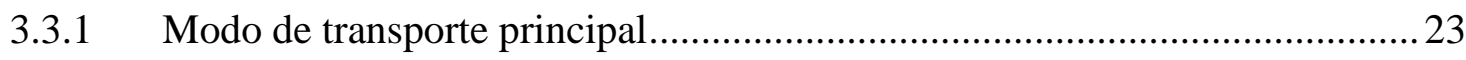

4 MODELO ECONOMETRICO ...................................................................... 25

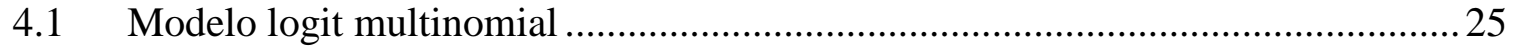




\section{UNiverSIDAD SANTO Tomas PRIMER CLAUSTRO UNIVERSITARIO DE COLOMBIA UNIDAD DE INVESTIGACIÓN}

4.1.1 Modo de transporte:.............................................................................25

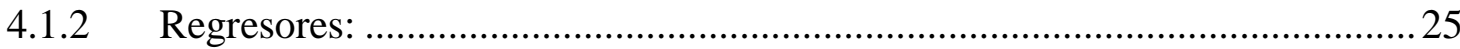

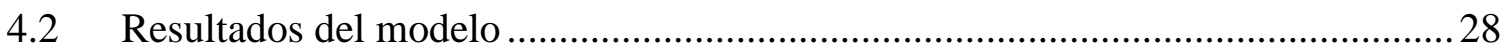

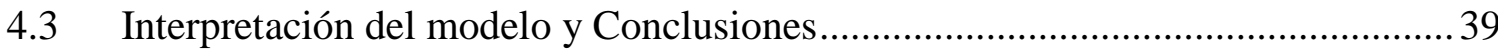

4.3.1 28.788 observaciones (Área Metropolitana del Valle de Aburrá) ...................39

4.3.2 2.594 observaciones (Barrios aledaños al sistema cable e individuos que

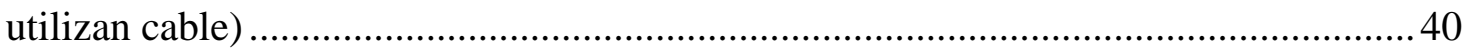

4.3.3 Conclusiones de la observación del Área Metropolitana con respecto a las viviendas y usuarios del sistema cable...................................................................... 41

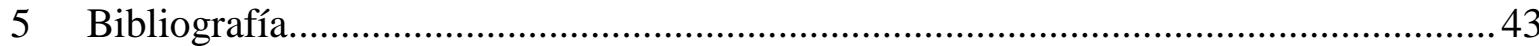




\section{㴽 UNIVERSIDAD SANTOTOMAS UNIDAD DE INVESTIGACIÓN}

\section{Lista de Tablas}

Tabla 1 Chi2 del modelo aplicado a las observaciones del Área Metropolitana.

Tabla 2 Chi2 del modelo aplicado a las observaciones de comunidades cercanas a estaciones de Cable y usuarios del modo Cable 28

Tabla 3 Rango de Edad del Área Metropolitana............................................................................................ 29

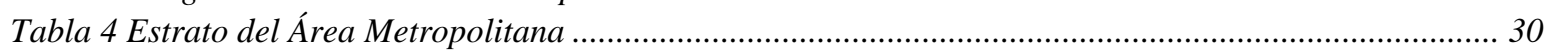

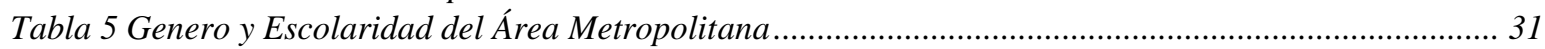

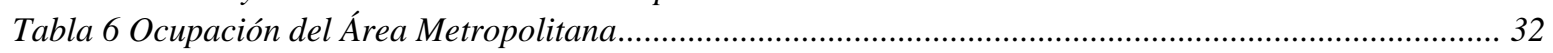

Tabla 7 Tenencia de Vivienda y Constante del modelo del Área Metropolitana ............................................... 33

Tabla 8 Rango de Edad de comunidades cercanas a estaciones y usuarios del modo Cable .......................... 34

Tabla 9 Estrato y Genero de comunidades cercanas a estaciones y usuarios del modo Cable ....................... 35

Tabla 10 Escolaridad de comunidades cercanas a estaciones y usuarios del modo Cable ............................. 36

Tabla 11 Ocupación de comunidades cercanas a estaciones y usuarios del modo Cable ................................ 37

Tabla 12 Tenencia de Vivienda y Constante del modelo de comunidades cercanas a estaciones y usuarios del modo Cable

\section{Lista de Ilustraciones}

Ilustración 1 Esquema de activos y estructuras de oportunidades de accesibilidad..................................... 18

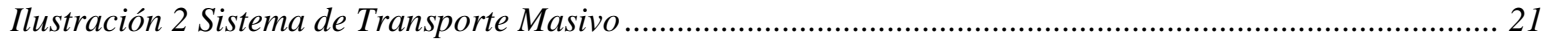

Ilustración 3 Sistema de bicicletas públicas......................................................................................... 22

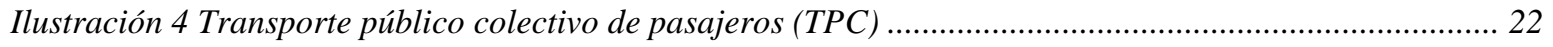




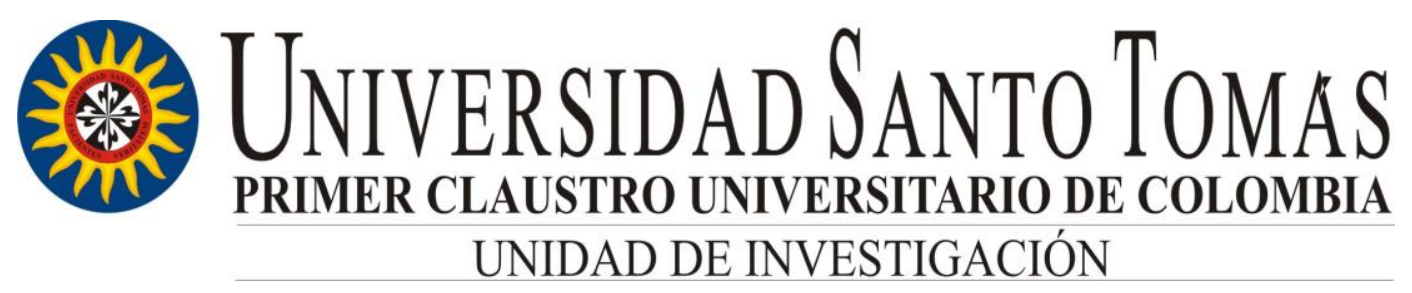

\section{INTRODUCCION}

Tal como lo define (McFadden, 1974), la demanda de viajes urbanos es el resultado de agregación sobre la población urbana, cada miembro está haciendo decisiones de viaje individuales basadas en sus necesidades personales y su entorno. Estas decisiones individuales son complejas, implican el propósito del viaje, la frecuencia, el tiempo, destino y modo de viaje. Además, estas opciones deben analizarse en el contexto de elecciones simultáneas de propiedad de automóviles, ubicación de vivienda y actividades de fin de viaje. Viajar normalmente no es un objetivo final del consumidor, sino más bien un concomitante de otras actividades como el trabajo, las compras y la recreación.

En el mundo, las ciudades que implementan servicios de transporte integrado y moderno generan el desarrollo de políticas públicas que conllevan a resultados positivos en términos económicos. Las principales evidencias empíricas demuestran un aumento en el empleo para ciudades que implementan transportes modernos e integrados como el metro, tal como sucedió en Estados Unidos, donde entre los años 1990 y 2004 las ciudades sin líneas de metro presentaron variación de $18.1 \%$, mientras que las ciudades con líneas de metro presentaron resultados de $35.8 \%$ de acuerdo al artículo de (Partridge, Rickman, Ali, \& Olfert, 2008).

En Santiago de Chile de acuerdo a un estudio de precios hedónicos de (Agostini \& Palmucci, 2008), se pudo evidenciar un aumento de precio en las viviendas que se encontraban en el recorrido del metro con respecto a zonas con similares características sin influencia de metro. En las Líneas 1, 2 y 5 se evidencio un aumento en el precio de 8.84\%, 27.16\%, y 6.72\% respectivamente. Así mismo se estableció que en algunas comunas como la de Santiago, los precios aumentaron en las líneas 1,2 y 5 un $16.9 \%, 10.2 \%$ y $11.6 \%$ en el mismo orden, aunque debe aclararse que dichas comparaciones se presentan en comunas con estratos altos.

Afirma (De Grange, 2010) que con las políticas de desarrollo urbano, la implementación de sistemas integrados de transporte mejoran la eficiencia de la economía, porque reducen los costos de viaje, y también se genera un mayor impacto en la actividad del comercio, aprovechando las economías de aglomeración y de igual manera, el impacto de la pobreza se reduce directamente tal como concluye, al estimar que el metro es el principal transporte de los más pobres.

El transporte público de Medellín, ha sido pionero en el modelo de transporte público urbano para Colombia, llevando una solución efectiva de movilidad, seguridad y cubrimiento geográfico. El sistema integrado metro ofrece el servicio metro, tranvía, metroplús, rutas 
integradas, bicicletas y metro cable, siendo este último el que mayor beneficio lleva a las comunidades más vulnerables.

El presente trabajo de investigación pretende demostrar a través de unos indicadores de bienestar, y algunas recomendaciones de la comisión (Stiglitz, Sen, \& Fitoussi, 2009), donde se pretende evaluar algunos aspectos de bienestar; el empleo u ocupación, escolaridad, propiedad de la vivienda y el modo de transporte, que a medida que avanza el desarrollo de la investigación y la evidencia empírica, se podrá determinar si estas variables fueron las adecuadas o si se hace necesario examinar otras opciones.

El indicador del empleo, en concordancia con los Objetivos de Desarrollo Sostenible (ODS) planteado por Naciones Unidas (Naciones Unidas, 2012), permiten observar de forma intrínseca dos objetivos: Objetivo 9 Industria, Innovación e Infraestructura: indica desarrollar infraestructura de calidad fiable y resilientes, promover la industrialización inclusiva y sostenible y fomentar la innovación, de este modo se busca fomentar el acceso equitativo y asequible para todos. Objetivo 11 Ciudades y Comunidades Sostenibles: plantea que, para poder continuar creciendo en el empleo y la prosperidad en las ciudades, se requiere superar problemas comunes de las ciudades como la congestión, la falta de fondos para prestar servicios básicos, la escasez de vivienda adecuada y el deterioro de la infraestructura, y el mismo objetivo plantea reducir la contaminación. Básicamente se plantea que el futuro deseado incluye ciudades con oportunidades, acceso a servicios básicos, energía vivienda, transporte y más facilidades para todos.

Las comunidades más vulnerables se ven obligadas a invadir predios en la periferia de las urbes, este fenómeno se ha presentado desde 1968, fecha en la cual se condenó la actividad de urbanización pirata tal como lo explica (Arango Z., 1981). De esta manera, la periferia genera grandes asentamientos urbanos que no cuentan con la infraestructura adecuada para tener unas condiciones de vida óptimas: riesgo, precariedad, improvisación sin espacio público y callejones con hacinamiento de pobladores.

Además de lo anterior, la falta de integración en la movilidad presenta problemas sociales y económicos que solo pueden ser resueltos si existe una verdadera unificación y facilidad de acceso y movilidad entre la periferia marginada y la urbe con oportunidades.

(Acemoglu \& Robinson, 2012), en su libro "Porque Fracasan los Países", afirma que cada sociedad funciona gracias a un conjunto de reglas políticas y económicas creadas e impuestas por el Estado y los ciudadanos colectivamente. Y mientras las instituciones económicas dan forma a los incentivos económicos (p. 59), es el proceso político lo que determina bajo qué instituciones económicas se vivirá, y son las instituciones políticas las que determinan cómo funciona ese proceso (p. 60). A modo de ejemplo, las instituciones políticas de una nación 
marcan la capacidad de los ciudadanos de controlar a los políticos e influir en su comportamiento.

Así, para Acemoglu y Robinson, en la vida real, las instituciones influyen en el comportamiento mediante incentivos. En el presente proyecto la institución se puede entender como el sistema de transporte que mejora el comportamiento y la calidad de vida de la comunidad que lo utiliza.

La decisión que tienen los individuos por las conductas sociales afecta directamente el comportamiento económico de una región, esto de acuerdo a las bases que fundamentan la economía institucional. De igual forma, la implementación de tecnología que vincula la racionalidad y la evolución del aprendizaje, se encuentra relacionada al sistema político y social. Por estas razones, una adecuada implementación de políticas públicas de inversión en infraestructura de transporte, puede modificar la conducta de las comunidades que se verá beneficiada con el desarrollo económico de una ciudad.

De esta manera puede darse una interpretación al pensamiento de Douglass North, puesto que, si los individuos dominantes dejan de lado el egoísmo, así como sus intereses particulares y dan prioridad al bienestar de las comunidades más vulnerables, los resultados benefician en mayor medida a los grandes grupos económicos que ven la oportunidad de crecimiento, jalonando directamente la economía de la región. Los individuos trabajadores y los empresarios ven una oportunidad de crecimiento cuando las condiciones de transporte son óptimas.

Con fundamento en esta problemática, se establece para el presente estudio de investigación, determinar cuál es la demanda de transporte de la ciudad de Medellin y su Área Metropolitana (siguiendo el modelo planteado por McFadden en 1974), así como establecer cuáles son las condiciones socio económicas de los ciudadanos encuestados que utilizan transporte público, establecer si la política pública de implementación de sistemas de transporte público aéreo (Metro Cable) ha aumentado el bienestar social, también se puede establecer cuál es el modo principal de transporte publico utilizado y a su vez, evaluar la probabilidad de utilización del sistema de transporte "Metro Cable" para las personas que habitan en los barrios aledaños a las estaciones.

De acuerdo a (Molina \& Fernández, 2013) los proyectos de infraestructura moderna tienen unos claros beneficios sociales y ambientales en las ciudades y por tanto, contribuyen al objetivo de desarrollo sostenible y accesibilidad planteado por las Naciones Unidas (2012), que aunque merecen significativas inversiones en infraestructura, los resultados a largo plazo son positivos. 


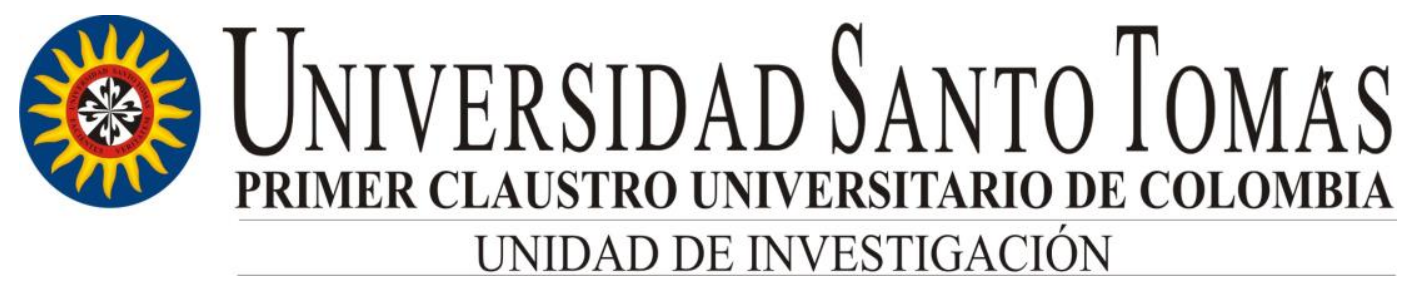

El aporte más importante que pretende dejar la presente investigación, es que puede servir como herramienta para la implementación de políticas públicas en las cuales prime el interés de disminución de la pobreza y reducción de la brecha de desigualdad a través de la medición de las condiciones socioeconómicas, la escolaridad y la tenencia de la propiedad. A nivel académico, la presente investigación se proyecta aportar un nuevo escenario que complementa dos ciencias como lo es la economía y la ingeniería civil, desde una perspectiva técnica multidisciplinaria que en escenarios recurrentes no se encuentran ligadas; el desarrollo económico y la infraestructura pero que en la realidad deben ser complementarias para tomar decisiones acertadas. 


\section{UNIVERSIDAD SANTO Tomas PRIMER CLAUSTRO UNIVERSITARIO DE COLOMBIA UNIDAD DE INVESTIGACIÓN}

\section{FUNDAMENTOS TEORICOS}

El modelo de demanda de transporte propuesto por Daniel McFadden (1974), pone de manifiesto la relación de la demanda agregada observada con la demanda individual, ya que en una población de consumidores que son homogéneos con respecto a los presupuestos enfrentados, la demanda agregada será igual a la individual y todas las variaciones sistemáticas en la demanda agregada se interpretan como generadas por una variación común en el margen intensivo de las mismas demandas individuales en ausencia de variaciones no observadas en los gustos o presupuestos.

Puesto que el objetivo central de la presente tesis pretende demostrar el impacto sobre el bienestar de los hogares en la utilización de sistemas de transporte publico moderno, y su incidencia en el nivel de empleo de las comunidades, en los barrios donde se implementan las líneas de metro cable en la ciudad de Medellín en el año 2017, será necesario identificar la corriente económica que servirá de marco a la investigación.

De esta manera se establece que el Neoinstitucionalismo es la base de la presente investigación, uno de sus mayores exponentes (North, 1993) realizó la principal contribución que fue introducir la idea de la relevancia del papel de las instituciones en el desempeño económico y el cambio institucional.

North define que el rol de la tecnología hoy estaría sobrevalorado puesto que el marco institucional global juega un rol significativo y afirma que las elecciones históricas están determinadas por el path dependence, simplemente por la naturaleza de las limitaciones provenientes del pasado. Este tipo de trayectorias se observan cuando el proceso depende de las decisiones tomadas por los actores y no solo por las condiciones del momento.

El transporte es clave para un desarrollo económico puesto que las comunidades pueden desplazarse; de aquí se derivan múltiples beneficios como el acceso a un empleo, a la educación, a la salud y el acceso a los mercados, de este modo los ciudadanos tienen mejor movilidad, lo cual ahorra tiempo y maximiza oportunidades. De acuerdo a (Maciulis, Vasilis, \& Jakubauskas, 2009) el transporte afecta el precio de los bienes, ya que puede disminuir o aumentar su oferta, se pueden formar cadenas de distribución y se atrae actividad donde aumenta la competitividad y el consumo a nivel microeconómico.

Las personas que tienen menor posibilidad de movilidad están en desventaja y corren un riesgo de exclusión social de acuerdo a (Currie \& Stanley, 2008). De igual forma lo define (Schonfelder \& Axhausen, 2003) al explicar que las personas que afrontan mayores distancias, tienen menos oportunidades y por tanto un mayor riesgo de exclusión social. 
De acuerdo al informe que se gestó en la Conferencia de las Naciones Unidas en Río de Janeiro en 2012 (Naciones Unidas, 2012), la agenda 2030 para el Desarrollo Sostenible se deben mejorar las políticas sociales y de empleo apropiadas para garantizar sociedades inclusivas y sostenibles y para alcanzar los Objetivos de Desarrollo Sostenible ODS.

El desarrollo económico en la implementación de sistemas de transporte moderno ha sido marcado por experiencias que permiten un análisis determinante en el desarrollo de este documento, puesto que son un referente para que el autor de este trabajo presente un análisis consecuente. Para ello es de vital importancia conocer la experiencia que se ha presentado, puesto que permitirá entender si de acuerdo al planteamiento del problema, los indicadores internacionales comparables en materia de bienestar van ligados de la mano de la implementación de transportes urbanos.

\subsection{Estado del arte}

El estudio realizado por (McFadden, 1974) determina que el sistema de transporte es un componente crítico de cada economía urbana, y que las decisiones de política de transporte pueden tener un profundo efecto sobre el desarrollo del sistema urbano. Los proyectos de transporte público son a menudo masivo y mutuamente excluyente, con efectos acumulativos irreversibles sobre periodos largos. Si se desea evitar grandes pérdidas sociales, una planificación cuidadosa basada en un un cálculo de costo-beneficio conceptualmente acertado y empíricamente preciso es esencial.

Los pronósticos precisos de la demanda de viajes bajo políticas de transporte alternativas son requeridos para cálculos precisos de beneficios. Para ser completamente satisfactorios, estos pronósticos deben ser lo suficientemente sensibles como para reflejar el impacto del cambio urbano y el entorno durante la vida útil de los proyectos de transporte propuestos (McFadden, 1974).

Christopher B. Leinberger, es un investigador y experto mundial, profesor de la Universidad de Michigan, que defiende el uso del sistema tranviario, manifiesta de una forma bastante agresiva, que aquellas ciudades que no implementen este tipo de sistemas de transporte están condenadas a seguir viviendo en el siglo 20. En un artículo (Leinberger, 2014) manifiesta que las líneas tranviarias son permanentes, lo que indica al mercado privado que el sistema de transporte no va a cambiar pronto; con lo cual el desarrollo económico financiado con fondos privados en torno a las estaciones de tranvía, tren ligero y Metrorail es muchísimo mayor que la inversión privada junto a las paradas de autobús. 
En cuanto a la elección de un determinado medio de transporte, (Fajardo Hoyos \& Gómez Sánchez, 2015) publicaron un estudio realizado en el año 2014, titulado "Análisis de la elección modal de transporte público y privado en la Ciudad de Popayán", donde realizan un análisis basándose en la teoría del consumidor, acerca de cuáles son los aspectos que influyen de manera directa en el proceso de elección del medio de transporte óptimo para la ciudad de Popayán, teniendo como resultado que factores tales como, los costos, los ingresos, la edad y el tiempo son capaces de explicar la elección de un medio de transporte en esa Ciudad con mayor precisión, frente a aspectos que no revisten un nivel de importancia dentro del modelo.

Patick M. Condon, (Condom, Gruenberger, \& Klaptocz, 2008) experto mundial en temas de movilidad, profesor de la University of British Columbia, en el año 2008 publicó un artículo titulado: "The Case for the Tram: Learning from Portland - Sustainability by Design". En este estudio, el autor concluye que la implementación de un sistema de transporte masivo tranvía, mejora la economía de los hogares, puesto que el costo por viaje se verá reducido con respecto al sistema de buses, afirma también que el clima de inversión de hogares con mayor densidad será mejor, determina que es posible mejorar el acceso de las personas y por supuesto no se equivoca el autor al plantear que esto conlleva a una mejor calidad de vida en los barrios. La primera línea de tranvía en Portland fue inaugurada en el año 2001, recientemente el 22 de septiembre del año 2012 inauguro otra línea que continua con la expansión de un sistema que presta un servicio adecuado, optimo y eficiente.

En su estudio (Mosquera Abadía, 2015), se observa un referente sobre la forma como la utilización del Sistema de Transporte MIO, puede llegar a ser excluyente por cuanto la preferencia por ese servicio está determinada por los ingresos de las personas, por su actividad económica e incluso por su participación y soporte social.

Por otra parte y ahondando en temas más económicos, se debe tener en cuenta el fenómeno de valorización, ya que al referirnos a una posible fuente de desarrollo económico por medio de la implementación de sistemas de transporte masivo es preciso citar estudios como el del Profesor (Estupiñan, 2011), experto en impactos en el uso del suelo, en el año 2011 publica un documento, cuya conclusión principal en este tema es que las obras para implementar sistemas de transporte masivo, representan altos niveles de inversión que a largo plazo se traducen en un incremento de los valores de las propiedades en las zonas cercanas a las vías intervenidas para tales fines, por tal razón se establece en esta investigación una posible fuente de desarrollo económico eficiente.

De acuerdo a (Keyes, Shmotkin, \& Ryff, 2002), se generan unos parámetros de comportamiento sociales que tienen relación con el Bienestar Social, puesto que subjetivamente cada individuo realiza una valoración del funcionamiento dentro de una 
sociedad, anteriormente (Keyes, Social Well-Being, 1998) ya había definido el Bienestar Social como la apreciación que las personas hacen de las circunstancias y el funcionamiento dentro de la sociedad, tomando en consideración lo subjetivo y lo social, e influyendo directamente en el bienestar y la salud

Para el caso de la infraestructura de transporte público en las ciudades y la implementación de sistemas de transporte, de acuerdo a (Urry, Shove, \& Cass, 2005) el acceso a bienes y oportunidades, tienen un impacto relevante en la calidad de vida de las personas puesto que se manifiestan y se reproducen estructuras sociales que se ven reflejadas en las características de movilidad habitual.

El desplazamiento en las ciudades y puntualmente la movilidad en transporte público, no es una decisión individual, puesto que se evidencian limitaciones estructurales muy fuertes, tal como lo describen (Massot \& Orfeuil, 2005). Puesto que la implementación de sistemas de transporte moderno en las grandes urbes, tiene como principal propósito la integración de las comunidades y la velocidad de desplazamiento, no deja de llamar la atención, el hecho de que en los estratos sociales mas altos no se tengan previstos dichos sistemas, y esto conlleva a que la movilidad como desarrollo económico de una sociedad no se implemente de forma equitativa tal como lo presenta (Kaufmann, Bergman, \& Joye, 2004).

Estudios sobre movilidad urbana, accesibilidad y pobreza; han identificado variables que pueden establecer la facilidad u obstaculizar el acceso al transporte público, tal como lo presentan (Flamm, Jemelin, \& Kaufmann, 2007), que específicamente al enfocarse en el transporte público resumen unas condiciones particulares como son las oportunidades de transporte, la ordenación institucional, los rasgos individuales y las dinámicas socio territoriales.

\subsection{Estructuras de oportunidades de movilidad}

Los hogares se enfrentan en su cotidianeidad a situaciones que colocan en riesgo el bienestar, esto se evidencia en una actualidad cambiante y la capacidad de mitigación de dichas circunstancias, que finalmente conllevan a tener que buscar una recuperación con una capacidad de respuesta inmediata, para ello (Moser, 1998) afirma que, conociendo el portafolio de activos y sus estrategias de utilización, es posible facilitar la intervención social para que los hogares pobres puedan utilizar sus activos de forma "productiva".

La demanda de transporte deviene de las necesidades de funcionamiento social en cuanto a circulación de personas y de bienes y servicios, por que se dice entonces que el transporte se 


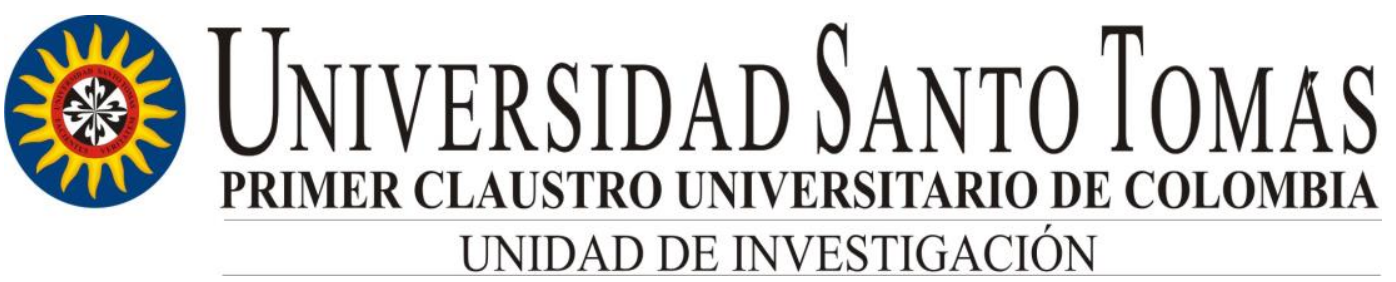

caracteriza, por lo que se denomina "demanda derivada" (Martinez, De Cristófaro, Sánchez, Hantke, \& Diaz, 2014)

La relación entre estos aspectos (Hernández, 2012) se presenta con el esquema de activos y estructuras de oportunidades de accesibilidad, puesto que es una orientación que se centra en la concepción de vulnerabilidad como una herramienta a la hora de analizar el bienestar de los hogares y las personas.

La accesibilidad es la interacción entre las oportunidades de desarrollo y las características de los hogares e individuos, que en el esquema se presenta como eje central. Entre estas variables se puede determinar que los hogares con ingresos bajos y sin vehículos propios, enfrentan la vulnerabilidad del transporte con un riesgo bastante alto. Es allí donde el Estado debe intervenir con una buena regulación y planificación de transporte público.

La movilidad también ha sido propuesta como el cubrimiento de necesidades de una comunidad mediante desplazamientos, puesto que permite la consecución de recursos económicos mayoritariamente en el mercado laboral (Ascher, 2005), a su vez en el mismo documento manifiesta como una necesidad del estado encontrar soluciones innovadoras para proporcionar transporte a los numerosos habitantes de las áreas periféricas de baja densidad que no tienen vehículos propios (niños, personas con movilidad reducida o de escasos recursos.

De acuerdo a (Cortés \& Figueroa , 2012) el intercambio modal en ciudades como Santiago de Chile, derivado de la implementación de Transantiago permitió la transformación del encadenamiento de desplazamientos en una estrategia de movilidad universal, posible de realizar por todos los habitantes de la ciudad. Esta universalización facilitó la proliferación de áreas comerciales en sectores históricamente desprovistos, facilitando la resolución de una serie de necesidades cotidianas. Estas áreas comerciales ganaron complejidad en la medida en que aumentaron los flujos de personas.

Pero no solamente la oportunidad de movilidad refleja la satisfacción de necesidades, sino que, se ha venido presentando un efecto denominado: Starbucks, este efecto fue estudiado por (Mcguckin \& Murakami, 1999) y los resultados indican que durante las últimas décadas, algunas de las comidas que las personas realizaban en el hogar fueron reemplazadas por comidas rápidas en medio de los viajes al trabajo o de regreso al hogar (especialmente el desayuno o la cena). 
Ilustración 1 Esquema de activos y estructuras de oportunidades de accesibilidad

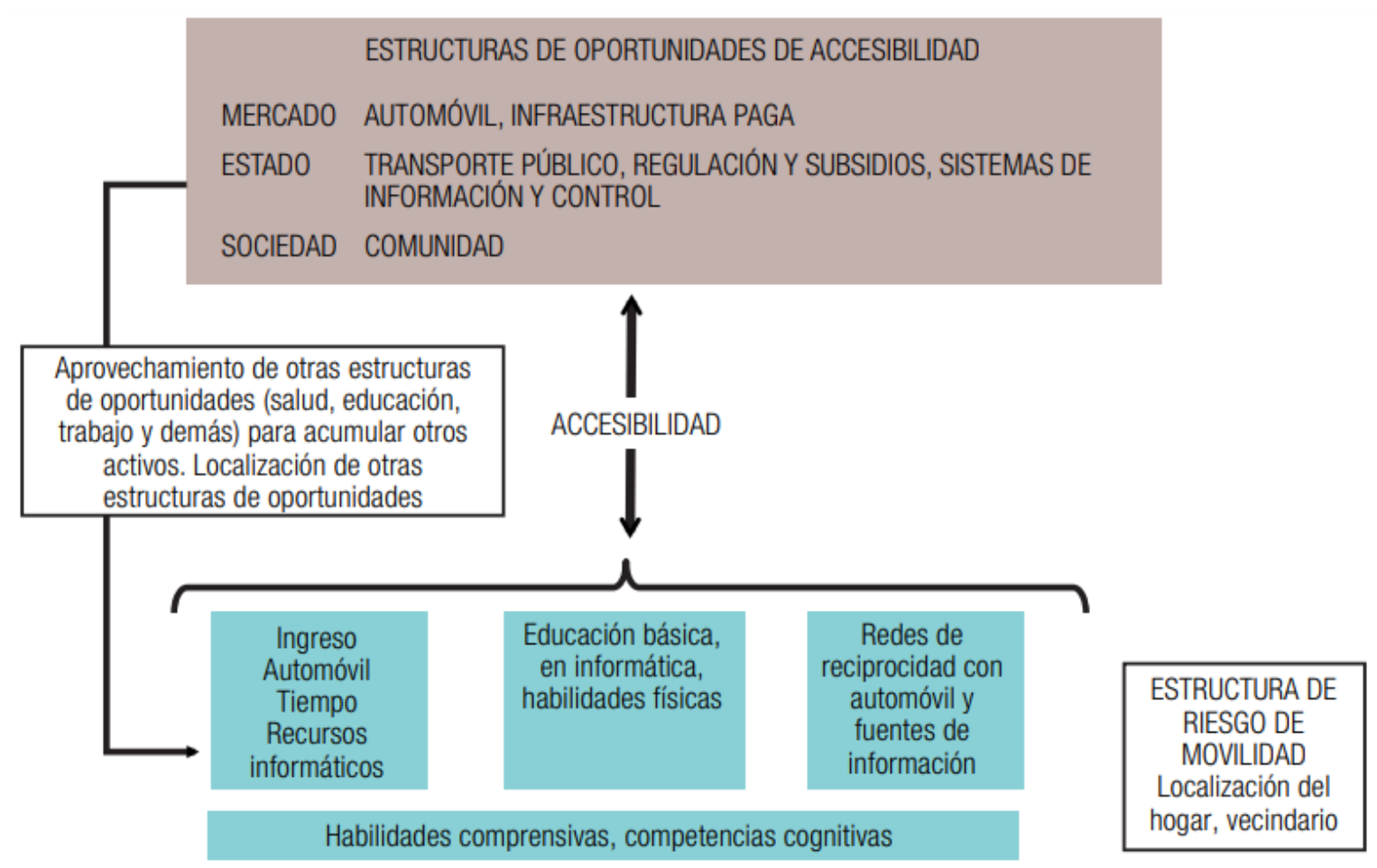

Fuente: Hernández, D. (2012). Activos y estructuras de oportunidades de movilidad. Una propuesta analítica para el estudio de la accesibilidad por transporte público, el bienestar y la equidad. EURE, 38(115), 117-135.

El enfoque Transit/Transport Oriented Development-TOD surge en el marco de lo que en Norteamérica se denominó growth management, administrar responsablemente el crecimiento urbano, y que desde finales de los años setenta del siglo XX aglutina el despliegue conjunto de herramientas dirigidas a controlar las transformaciones territoriales buscando el difícil compromiso entre crecimiento económico, equidad social y sostenibilidad ambiental. No es simple lucha contra el sprawl; por ello, es en este contexto en el que la importancia de las infraestructuras de transporte en la configuración urbana adquiere nuevo significado, a la vez que se buscan caminos para integrarlas en un proyecto sostenible de ciudad tal como lo define (Santos y Ganges \& de las Rivas Sanz, 2008). 


\section{UNIVERSIDAD SANTO TOMAS PRIMER CLAUSTRO UNIVERSITARIO DE COLOMBIA UNIDAD DE INVESTIGACIÓN}

\subsection{Estudios de transporte en oportunidades económicas}

La disminución de la pobreza se relaciona con el transporte, puesto que aumenta las oportunidades del mercado laboral, y este es un razonamiento que debería perseguir proyectos de infraestructura en el transporte público, ya que incrementa la competitividad y el desarrollo económico tal como lo plantea (Davies, Banister, \& Hall, 2004).

Se presentan aquí dos oportunidades de estudio, el primero en la ubicación sectorial de las empresas, y el segundo los beneficios de aglomeración vinculados a la inversión del transporte; este análisis realizado por (Boarnet \& Geho, 2005), compara regiones o áreas metropolitanas, generando patrones espaciales de crecimiento del empleo.

La inversión en sistemas de transporte, genera diversificación y fomenta la creación de nuevas empresas, tal como lo plantea (Holl, 2004), también genera desarrollo económico al comprobarse el efecto del incentivo de los impuestos en una distribución espacial y el retorno de la inversión como lo presenta (Fisher \& Peters, 1998). Otra manera de estimar el crecimiento en el mercado laboral en una distribución espacial es calcular y medir el número de trabajos ofrecidos en las zonas empresariales utilizando una función potencial, tal como lo presenta (Boarnet M. , 1994).

Varios estudios permiten establecer el efecto de la implementación de proyectos de transporte con respecto a la ubicación de las zonas empresariales, sin embargo, algunos de ellos no contemplan los cambios en la oferta de trabajo, para ser más acertados en los resultados, puede establecerse dos ecuaciones de población y crecimiento económico, tal como lo presentado por (Carlino \& Mills, 1987). 


\section{Universidad SANTOTOMaS PRIMER CLAUSTRO UNIVERSITARIO DE COLOMBIA UNIDAD DE INVESTIGACIÓN}

\section{CARACTERIZACIÓN DEL TRANSPORTE PUBLICO EN MEDELLIN}

El desarrollo de la infraestructura de transporte público en Medellín se planifica como un área metropolitana, en la cual se integran nueve municipios adicionales: Barbosa, Bello, Caldas, Copacabana, Envigado, Girardota, Itagüí, La Estrella y Sabaneta.

Este sistema es conocido como Sistema Integrado de Transporte del Valle de Aburrá - SITVA que es el conjunto de modos de transporte público que permite a los ciudadanos metropolitanos desplazarse por los diez municipios del Valle de Aburrá y está conformado por varios subsistemas (Área Metropolitana Valle de Aburrá, 2019):

\subsection{Subsistema de transporte masivo:}

Conformado por el metro, los cables, el tranvía y los buses que circulan por carriles preferenciales y exclusivos (Bus de Transito Rápido BTR), así como sus servicios alimentadores.

\subsubsection{El Metro:}

El Valle de Aburrá cuenta con 31,3 kilómetros de línea ferroviaria que mueve 800.000 personas diarias a una velocidad promedio de $37 \mathrm{~km} / \mathrm{h}$. El metro inició su operación en 1995, hoy cuenta con dos líneas, 27 paradas y una flota de 80 unidades de tren.

\subsubsection{Los Cables}

Las líneas de cables se extienden para subir las laderas de Medellín y uno de ellos se prolonga en plan turístico hasta el Parque Arví. En total son 11,9 km de cables con 12 paradas repartidas en cinco líneas y una flota de 362 telecabinas que moviliza a diario 41.000 pasajeros. El primer cable de la ciudad fue puesto en servicio en el 2004.

\subsubsection{El Tranvía}

Inició su operación en el 2016, presta servicio con una única línea de $4.3 \mathrm{~km}$ y nueve paradas, cuenta con una flota conformada por doce tranvías que movilizan 45.000 pasajeros diarios. El acompañamiento social en su fase de construcción permitió convertir el corredor del tranvía en galería urbana, un recorrido con memoria que permite hoy ver además del paisaje de la ladera oriental, los murales y la gastronomía local. 


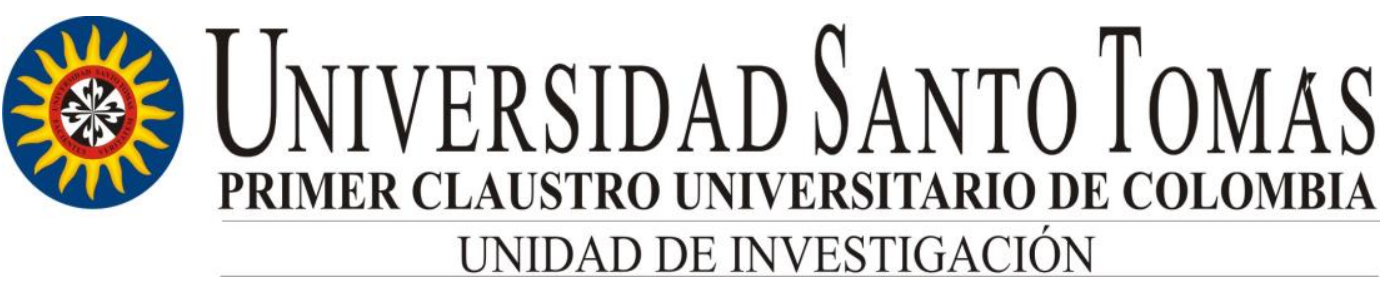

\subsubsection{El Metroplús}

Moviliza a diario 125.000 pasajeros en dos líneas que suman $26 \mathrm{~km}$ de recorrido, cuenta con 48 paradas atendidas por una flota de 30 buses articulados y 47 padrones. Desde el inicio de su operación en el 2011, ha sido evidente la eficiencia de los viajes del Metroplús por los carriles exclusivos y la próxima conexión con los sistemas de Envigado e Itagüí son un paso más hacia la completa integración del sistema.

\subsubsection{Buses alimentadores y rutas integradas}

Organizados por cuencas, completan el sistema que entiende cómo se mueve la gente en el Valle de Aburrá y facilita sus desplazamientos en términos tarifarios y de eficiencia. En el sistema se movilizan a diario 110.000 personas, en 35 rutas operadas por una flota de 302 buses de 40 pasajeros y 65 buses de 19 pasajeros que conectan 1.033 paraderos en el Valle de Aburrá.

Ilustración 2 Sistema de Transporte Masivo

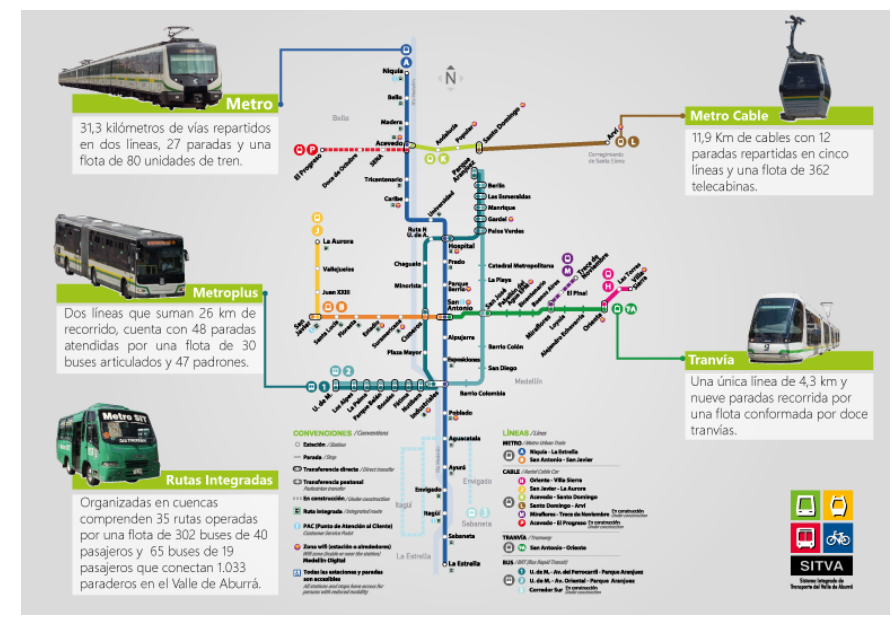

Fuente: Área Metropolitana Valle de Aburrá

2.2 Subsistema de transporte público colectivo de pasajeros (TPC):

Conformado por las rutas de buses con un radio de acción municipal y metropolitano.

2.3 Sistema de bicicletas públicas del Valle de Aburrá - Encicla. 


\section{Universidad SANTOTOMas PRIMER CLAUSTRO UNIVERSITARIO DE COLOMBIA UNIDAD DE INVESTIGACIÓN}

Ilustración 3 Sistema de bicicletas públicas

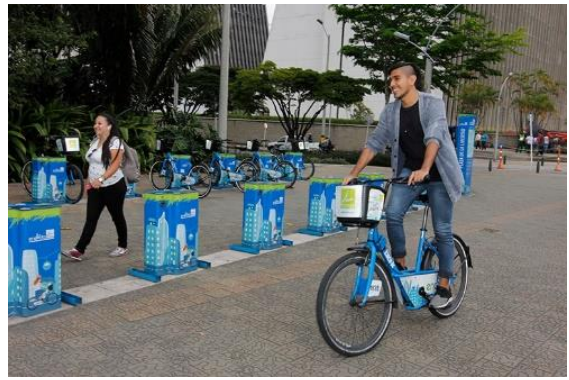

Fuente: Área Metropolitana Valle de Aburrá

Ilustración 4 Transporte público colectivo de pasajeros (TPC)

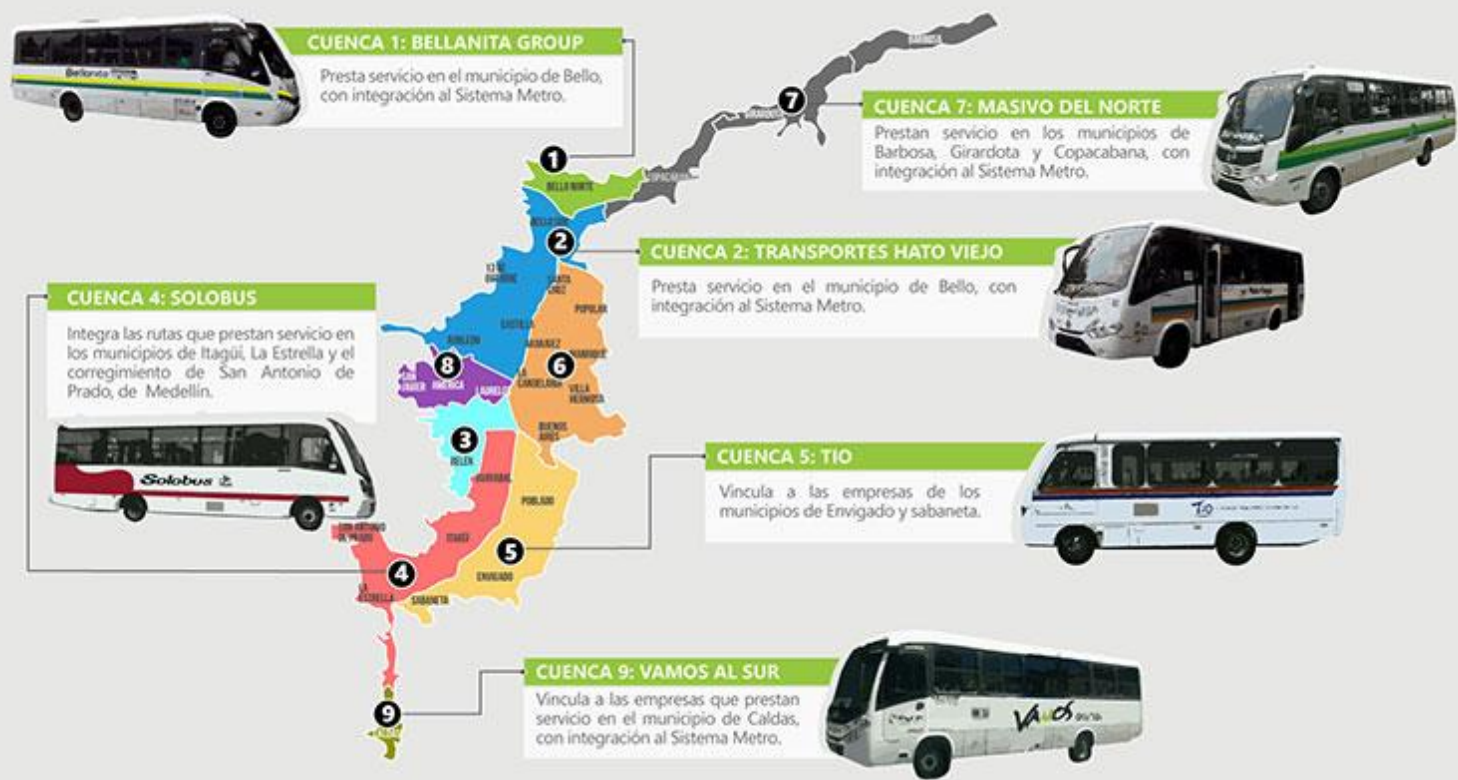

Fuente: Área Metropolitana Valle de Aburrá 


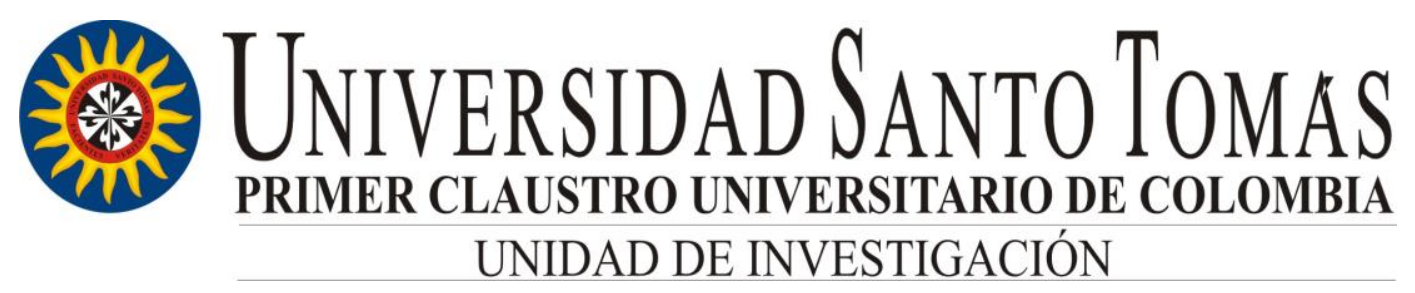

\section{METODOLOGIA Y FUENTES DE INFORMACIÓN}

\subsection{Data cleansing}

El muestreo estadístico de la encuesta origen-destino 2017 se configuro mediante muestreo sistemático en las zonas urbanas y en las macrozonas rurales de forma aleatoria en los sectores más poblados. La encuesta se realizó a 16.340 hogares obteniendo 87.613 datos de viajes. La primera depuración se filtro retirando de la muestra aquellos datos cuyo origen de municipio se encontraban fuera del Área Metropolitana del Valle de Aburra.

$\mathrm{Al}$ cruzar las bases de datos de los hogares, moradores y viajes, se evidencio que existían encuestas que no contaban con información relevante para el objeto de la presente investigación como el caso del estrato e identificación de los barrios, así mismo se toma la decisión de depurar a una sola encuesta por individuo, es decir solo los datos con respecto al origen desde el lugar del hogar encuestado y no el dato del viaje de retorno, finalmente se obtienen 28.788 datos.

\subsection{Consideraciones éticas y recolección de datos}

La información base es de carácter público, se encuentra disponible para quienes deseen conocer los datos, y también se contó con una colaboración y soporte permanente por parte de funcionarios del Área Metropolitana Valle de Aburrá.

\subsection{Variables explicativas}

\subsubsection{Modo de transporte principal}

La EOD presenta siete trayectos de movilidad posible para cada individuo, estos trayectos tienen cada uno 42 opciones dependiendo de la etapa del trayecto en que se encuentre. Un modo de transporte es caminar, otro modo es cable, también lo es el metro, así como la moto o el vehículo particular, etcétera. Un ejemplo es el de un ciudadano que camina tres cuadras desde su hogar, ingresa al cable, continua su trayecto con el metro, camina una cuadra para utilizar el Metroplús, después utiliza la ruta integrada y finalmente camina tres cuadras para llegar a su destino; en este ejemplo, el ciudadano realizo 7 trayectos con 6 modos diferentes, puesto que caminar 3 cuadras se repite en dos ocasiones.

Con los datos de viajes de la EOD, los ciudadanos que utilizan un solo trayecto en su desplazamiento, ya no presentan información disponible en el segundo trayecto. La mayor 
cantidad de registros se evidencia en el segundo trayecto, razón por la cual se opta por utilizar esta variable, pero reduciendo las opciones a tan solo 9, es decir: caminar, independientemente del número de cuadras es un solo modo de transporte, igualmente utilizar un vehículo particular independientemente de si se es el conductor o el acompañante, es un solo modo de transporte, etcétera.

\subsubsection{Modo de transporte}

Las categorías de modos de transporte se categorizaron en 8 opciones; metro, sistema integrado (incluye ruta integrada, metroplús y tranvía), bus (incluye urbano e intermunicipal), cable, taxi (incluye taxi individual, taxi colectivo y taxi intermunicipal), auto particular (incluye conductores y/o acompañantes), moto (incluye conductores y/o acompañantes) y a pie (independientemente de la distancia recorrida).

\subsubsection{Ocupación}

La ocupación contaba inicialmente con 12 categorías, se reducen las variables y se obtienen 5 categorías: desempleado, ama de casa, estudiante, trabajador y jubilado.

\subsubsection{Escolaridad}

Esta variable también fue categorizada, reduciendo las opciones de 10 categorías a 4, quedando establecidas de la siguiente forma: primaria, educación no formal, bachillerato y universitario.

\subsubsection{Rango de Edad}

Se categorizan los rangos de edad, entre 15 y 24 años, entre 25 y 34 años, entre 35 y 44 años, entre 45 y 54 años y mayor de 55 años de edad.

\subsubsection{Estrato}

La estratificación continua con los mimos datos de la EOD

\subsubsection{Genero}

Se categoriza en dos descripciones 0 para hombre y 1 para mujer.

\subsubsection{Tenencia de la vivienda}

La propiedad de la vivienda se reduce de cuatro opciones a solamente dos; 0 para vivienda propia, 1 para otros (incluye alquilada, proporcionada por otros y no sabe o no responde)

\subsubsection{Constante}

El software STATA automáticamente da valor a una constante. 


\section{Universidad SANTOTOMas PRIMER CLAUSTRO UNIVERSITARIO DE COLOMBIA UNIDAD DE INVESTIGACIÓN}

\section{MODELO ECONOMETRICO}

Algunos estudios como los de (Hensher, 1986) y (McFadden, 1984) han utilizado modelos de elección múltiple en los cuales no puede utilizarse el modelo probit multivariante, puesto que en dicho modelo los individuos tienen que tomar varias decisiones, cada una de ellas entre dos alternativas (Greene W. , 1998). Para el análisis de la presente investigación se analizará una sola decisión entre varias alternativas.

\subsection{Modelo logit multinomial}

La metodología Multinomial Logit (MNLM) es aplicada cuando la variable dependiente es categórica, pero estas categorías no tienen un orden. Esta metodología puede ser pensada como una estimación simultánea de modelos logit binarios para todas las comparaciones posibles de las categorías de resultados (Vásquez, 2008).

Los datos contienen la siguiente información de la muestra:

\subsubsection{Modo de transporte:}

$1=$ Metro

$2=$ Sistema Integrado

$3=$ Bus

$4=$ Cable

$5=$ Taxi

$6=$ Auto particular

$7=$ Moto

$8=$ A pie

\subsubsection{Regresores:}

Rango de edad, estrato, genero, escolaridad, ocupación, propiedad de la vivienda.

Siguiendo el análisis econométrico de (Greene, 1998) se establece que la elección del medio de transporte en la EOD es no ordenada, y al ser un modelo de elección múltiple puede venir generado por un modelo de utilidad aleatoria. Cuando el individuo iésimo ha de utilizar entre $J$ posibilidades, la utilidad de escoger la jésima opción es:

$$
U_{i j}=\beta^{\prime} z_{i j}+\epsilon_{i j}
$$


Cuando el ciudadano utiliza la opción jésima es porque, de entre las $J$ utilidades diferentes, la máxima es $U_{i j}$ el modelo estadístico se construye entonces teniendo en cuenta que escoger la alternativa jésima equivale a decir que

$$
\operatorname{Prob}\left(U_{i j}>U_{i k}\right) \text { para cualquier otro } k \neq j
$$

Para obtener la formulación concreta del modelo se escoge una función de distribución específica para los errores. Un ciudadano que utiliza una opción de transporte y tiene $\mathbf{x}_{\mathrm{i}}$ características individuales, tendrá unas ecuaciones estimadas que presentan un conjunto de probabilidades para las $J+1$ alternativas. El modelo presenta una indeterminación, ya que si se definen $\boldsymbol{\beta}_{\boldsymbol{j}}{ }^{*}=\boldsymbol{\beta}_{\boldsymbol{j}}+\boldsymbol{q}$ para cualquier vector $\boldsymbol{q}$ se obtienen las mismas probabilidades, puesto que todos los términos a los $\boldsymbol{q}$ afecta se cancelan. Para normalizar el modelo se define $\boldsymbol{\beta}_{\mathbf{0}}=\mathbf{0}$ y las probabilidades resultantes son:

$$
\begin{aligned}
& \operatorname{Prob}(Y=j)=\frac{e^{\beta^{\prime}{ }_{j} x_{i}}}{1+\sum_{k=1}^{J} e^{\beta^{\prime}{ }_{k} x_{i}}} \operatorname{para} j=1,2, \ldots J, \\
& \operatorname{Prob}(Y=0)=\frac{1}{1+\sum_{k=1}^{J} e^{\beta^{\prime}{ }_{k} x_{i}}}
\end{aligned}
$$

Ahora bien, en el modelo del software stata, como lo describe (Greene W. , 2012) vamos a suponer que hay $\mathbf{k}$ resultados categóricos $\mathbf{y}$, sin pérdida de generalidad, dejamos que la base resultado sea 1. La probabilidad de que la respuesta para la jésima observación sea igual al iésimo resultado es:

$$
p_{i j}=\operatorname{Pr}\left(y_{j}=i\right)=\left\{\begin{array}{l}
\frac{1}{1+\sum_{m=2}^{k} \exp \left(x_{j} \beta_{m}\right)}, \text { si } i=1 \\
\frac{\exp \left(x_{j} \beta_{i}\right)}{1+\sum_{m=2}^{K} \exp \left(x_{j} \beta_{m}\right)}, \text { si } i>1
\end{array}\right.
$$


donde $\mathbf{x}_{\mathbf{j}}$ es el vector fila de valores observados de las variables independientes para la observación $j$ y $\boldsymbol{\beta}_{\mathrm{m}}$ es el vector de coeficiente para el resultado $\boldsymbol{m}$. El log likelihood es:

$$
\ln L=\sum_{j} w_{j} \sum_{i=1}^{k} I_{i}\left(y_{j}\right) \ln P_{i k}
$$

Donde $\mathrm{w}_{\mathrm{j}}$ es un peso opcional $\mathrm{y}$

$$
I_{i}\left(y_{j}\right)=\left\{\begin{array}{c}
1, \text { si } y_{j}=i \\
0, \text { de otra manera }
\end{array}\right.
$$

Se usa la probabilidad máxima de Newton-Raphson para ecuaciones restringidas, el conjunto de restricciones está ortogonalizado y un subconjunto de parámetros maximizables seleccionados. Por ejemplo, un parámetro restringido a cero no es un parámetro maximizable. Si dos parámetros están restringidos para ser iguales entre sí, solo uno es un parametro maximizable.

Sea $\mathbf{r}$ el vector de parámetros maximizables. $\mathbf{r}$ es físicamente un subconjunto de los parámetros de la solución $\mathbf{b}$. Una matriz $\mathbf{T}$, y un vector $\mathbf{m}$, se define como:

$$
\mathrm{b}=\operatorname{Tr}+\mathrm{m}
$$

Entonces:

$$
\begin{gathered}
\frac{\partial f}{\partial \mathbf{b}}=\frac{\partial f}{\partial \mathbf{r}} \mathbf{T}^{\prime} \\
\frac{\partial^{2} f}{\partial \mathbf{b}^{\mathbf{2}}}=\mathbf{T} \frac{\partial^{2} f}{\partial \mathbf{r}^{2}} \mathbf{T}^{\prime}
\end{gathered}
$$

Donde $\mathbf{T}$ consiste en una forma de bloque en la que una parte es una permutación de la matriz de identidad y la otra parte describe cómo calcular los parámetros restringidos a partir de los parámetros maximizables.

Al ser el modelo Logit Multinomial un modelo econométrico, se presenta como una aproximación a la realidad esperada; las variables regresores pueden presentar variación de acuerdo a las condiciones de cada ciudad, los sistemas de transporte implementados, la topografía urbana y las políticas de subsidios al transporte reales. 


\section{Universidad SANTOTOMas PRIMER CLAUSTRO UNIVERSITARIO DE COLOMBIA UNIDAD DE INVESTIGACIÓN}

\subsection{Resultados del modelo}

Tabla 1 Chi2 del modelo aplicado a las observaciones del Área Metropolitana

\begin{tabular}{|l|r|r|r|}
\hline Multinomial logistic regression & Number of obs & $=$ & 28,788 \\
\cline { 2 - 4 } & LR chi2 $(168)$ & $=$ & 14647.44 \\
\cline { 2 - 4 } & Prob $>$ chi2 & $=$ & 0.0000 \\
\cline { 2 - 4 } Log likelihood $=-46028.394$ & Pseudo R2 & $=$ & 0.1373 \\
\hline
\end{tabular}

Tabla 2 Chi2 del modelo aplicado a las observaciones de comunidades cercanas a estaciones de Cable y usuarios del modo Cable

\begin{tabular}{|l|r|r|r|}
\hline Multinomial logistic regression & Number of obs & $=$ & 2,594 \\
\cline { 2 - 4 } & LR chi2(152) & $=$ & 1392.94 \\
\cline { 2 - 4 } Log likelihood $=-3965.5634$ & Prob > chi2 & $=$ & 0.0000 \\
\cline { 2 - 4 } & Pseudo R2 & $=$ & 0.1494 \\
\hline
\end{tabular}




\section{UNiversidad SANTOTomas

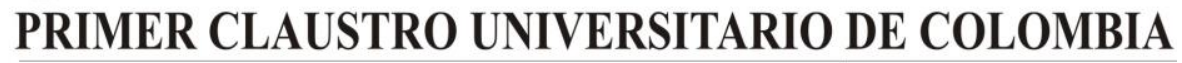 UNIDAD DE INVESTIGACIÓN}

Tabla 3 Rango de Edad del Área Metropolitana

MODO de TRANSPORTE

\begin{tabular}{|c|c|c|c|c|c|c|c|c|c|c|c|c|c|c|c|c|}
\hline \multirow[b]{2}{*}{ RANGO_EDAD } & \multicolumn{2}{|c|}{ Metro } & \multicolumn{2}{|l|}{ SIT } & \multicolumn{2}{|l|}{ Bus } & \multicolumn{2}{|c|}{ Cable } & \multicolumn{2}{|l|}{ Taxi } & \multicolumn{2}{|c|}{$\begin{array}{c}\text { Auto } \\
\text { Particular }\end{array}$} & \multicolumn{2}{|l|}{ Moto } & \multicolumn{2}{|l|}{ A pie } \\
\hline & & $\mathbf{P}>\mathbf{Z}$ & & $\mathbf{P}>\mathbf{Z}$ & & $\mathbf{P}>\mathbf{Z}$ & & $\mathbf{P}>\mathbf{Z}$ & & $\mathbf{P}>\mathbf{Z}$ & & $\mathbf{P}>\mathbf{Z}$ & & $\mathbf{P}>\mathbf{Z}$ & & $\mathbf{P}>\mathbf{z}$ \\
\hline \multicolumn{17}{|l|}{ Entre 15 y 24 años } \\
\hline Coef. & 3.130 .187 & $* * *$ & 2.251 .272 & $* * *$ & 2.087 .718 & $* * *$ & 3.815 .178 & $* * *$ & .8997366 & $* * *$ & -.2214606 & $*$ & 1.678 .075 & $* * *$ & -.5522136 & $* * *$ \\
\hline Std. Err. & .2835277 & & .1293847 & & .0908 & & .4799711 & & .1830972 & & .118786 & & .1151551 & & .0976976 & \\
\hline \multicolumn{17}{|l|}{ Entre 25 y 34 años } \\
\hline Coef. & 3.358 .367 & $* * *$ & 2.476 .928 & $* * *$ & 2.383 .913 & $* * *$ & 392.876 & $* * *$ & 1.573 .419 & $* * *$ & .6703394 & $* * *$ & 2.377 .248 & $* * *$ & -.3520719 & $* *$ \\
\hline Std. Err. & .2917542 & & .1389662 & & .100718 & & .5005174 & & .1958479 & & .12986 & & .1224343 & & .1326123 & \\
\hline \multicolumn{17}{|l|}{ Entre 35 y 44 años } \\
\hline Coef. & 3.287 .129 & $* * *$ & 2.245 .458 & $* * *$ & 2.224 .308 & $* * *$ & 3.984 .382 & $* * *$ & 1.426 .392 & $* * *$ & .9438347 & $* * *$ & 197.806 & $* * *$ & -.550001 & $* * *$ \\
\hline Std. Err. & .2934655 & & .1417361 & & .1028624 & & .5001345 & & .201479 & & .1313212 & & .1252555 & & .1421404 & \\
\hline \multicolumn{17}{|l|}{ Entre 45 y 54 años } \\
\hline Coef. & 3.042 .624 & $* * *$ & 2.155 .006 & $* * *$ & 2.189 .172 & $* * *$ & 3.494 .353 & $* * *$ & 1.411 .977 & $* * *$ & .7607031 & $* * *$ & 1.357 .949 & $* * *$ & -.4602215 & $* *$ \\
\hline Std. Err. & .2944312 & & .141295 & & .1017509 & & .5087405 & & .1975548 & & .1308519 & & .1283488 & & .1346917 & \\
\hline \multicolumn{17}{|l|}{ Mayor de 55 años } \\
\hline Coef. & 2.697 .904 & $* * *$ & 1.721 .449 & $* * *$ & 1.924 .187 & $* * *$ & 2.907 .295 & $* * *$ & 146.194 & $* * *$ & .2928883 & $* *$ & -.2327509 & $*$ & -1.075 .625 & $* * *$ \\
\hline Std. Err. & .294473 & & .1403483 & & .0987589 & & .508402 & & .1868526 & & .1299714 & & .1498084 & & .13951 & \\
\hline
\end{tabular}

Nota: * No significativo

** $\quad$ Entre $95 \%$ y $99 \%$

$* * *>$ a $99 \%$ 


\section{Universidad SantoTomas

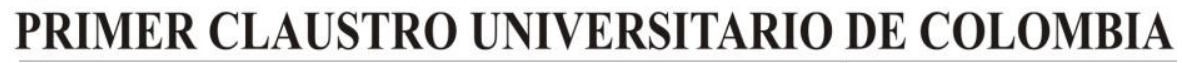 UNIDAD DE INVESTIGACIÓN}

Tabla 4 Estrato del Área Metropolitana

MODO de TRANSPORTE

\begin{tabular}{|c|c|c|c|c|c|c|c|c|c|c|c|c|c|c|c|c|}
\hline \multirow[b]{2}{*}{ ESTRATO } & \multicolumn{2}{|c|}{ Metro } & \multicolumn{2}{|l|}{ SIT } & \multicolumn{2}{|l|}{ Bus } & \multicolumn{2}{|l|}{ Cable } & \multicolumn{2}{|l|}{ Taxi } & \multicolumn{2}{|c|}{$\begin{array}{c}\text { Auto } \\
\text { Particular }\end{array}$} & \multicolumn{2}{|c|}{ Moto } & \multicolumn{2}{|l|}{ A pie } \\
\hline & & $\mathbf{P}>\mathbf{Z}$ & & $\mathbf{P}>\mathbf{Z}$ & & $\mathbf{P}>\mathbf{Z}$ & & $\mathbf{P}>\mathbf{Z}$ & & $\mathbf{P}>\mathbf{Z}$ & & $\mathbf{P}>\mathbf{Z}$ & & $\mathbf{P}>\mathbf{Z}$ & & $\mathbf{P}>\mathbf{Z}$ \\
\hline \multicolumn{17}{|l|}{ ESTRATO 2} \\
\hline Coef. & .0292621 & $*$ & -.0077223 & $*$ & -.0578025 & $*$ & -.4827076 & $* *$ & .1634262 & $*$ & .3602075 & $* *$ & .0396022 & $*$ & .4762964 & $* * *$ \\
\hline Std. Err. & .1038913 & & .0679368 & & .0545894 & & .1468126 & & .1374665 & & .1188299 & & .068643 & & .1002133 & \\
\hline \multicolumn{17}{|l|}{ ESTRATO 3} \\
\hline Coef. & .4625773 & $* * *$ & -.0749976 & $*$ & -.008598 & $*$ & -232.152 & $* * *$ & .7629234 & $* * *$ & 1.290 .178 & $* * *$ & .1951563 & $* *$ & .9253448 & $* * *$ \\
\hline Std. Err. & .1060177 & & .0742147 & & .0589854 & & .2985043 & & .1376247 & & .1165561 & & .0736234 & & .1034928 & \\
\hline \multicolumn{17}{|l|}{ ESTRATO 4} \\
\hline Coef. & .4485927 & $* *$ & -.6862438 & $* * *$ & -.1283893 & $*$ & -3.689 .419 & $* * *$ & 1.365 .448 & $* * *$ & 2.057 .113 & $* * *$ & .0862745 & $*$ & 1.360 .249 & $* * *$ \\
\hline Std. Err. & .134002 & & .1191077 & & .0830002 & & 1.011 .404 & & .1551874 & & .1266291 & & .1031011 & & .128164 & \\
\hline \multicolumn{17}{|l|}{ ESTRATO 5} \\
\hline Coef. & .4711095 & $* *$ & -.6389766 & $* * *$ & -.2929431 & $* *$ & -16.169 & $*$ & 1.542 .214 & $* * *$ & 3.037 .568 & $* * *$ & .1226075 & $*$ & 224.037 & $* * *$ \\
\hline Std. Err. & .1642842 & & .155797 & & .1122126 & & 7.312 .323 & & .1749383 & & .1326223 & & .1324119 & & .1361428 & \\
\hline \multicolumn{17}{|l|}{ ESTRATO 6} \\
\hline Coef. & .898424 & $* *$ & -.8719867 & $* *$ & -.2987868 & $*$ & -1.761 .837 & $*$ & 2.544 .199 & $* * *$ & 4.722 .899 & $* * *$ & .4284972 & $*$ & 3.083 .807 & $* * *$ \\
\hline Std. Err. & .3280356 & & .4436817 & & .2841199 & & 4.319 .071 & & .2709023 & & .2006052 & & .2904298 & & .2277748 & \\
\hline
\end{tabular}

Nota: * No significativo

** Entre $95 \%$ y $99 \%$

$* * *>$ a $99 \%$ 


\section{UNiversidad SANTOTomas PRIMER CLAUSTRO UNIVERSITARIO DE COLOMBIA UNIDAD DE INVESTIGACIÓN}

Tabla 5 Genero y Escolaridad del Área Metropolitana

MODO de TRANSPORTE

\begin{tabular}{|c|c|c|c|c|c|c|c|c|c|c|c|c|c|c|c|c|}
\hline \multirow[b]{2}{*}{ GENERO } & \multicolumn{2}{|c|}{ Metro } & \multicolumn{2}{|l|}{ SIT } & \multicolumn{2}{|l|}{ Bus } & \multicolumn{2}{|l|}{ Cable } & \multicolumn{2}{|l|}{ Taxi } & \multicolumn{2}{|c|}{$\begin{array}{c}\text { Auto } \\
\text { Particular }\end{array}$} & \multicolumn{2}{|l|}{ Moto } & \multicolumn{2}{|l|}{ A pie } \\
\hline & & $\mathbf{P}>\mathbf{z}$ & & $\mathbf{P}>\mathbf{z}$ & & $\mathbf{P}>\mathbf{Z}$ & & $\mathbf{P}>\mathbf{z}$ & & $\mathbf{P}>\mathbf{z}$ & & $\mathbf{P}>\mathbf{z}$ & & $\mathbf{P}>\mathbf{z}$ & & $\mathbf{P}>\mathbf{Z}$ \\
\hline \multicolumn{17}{|l|}{ Mujer } \\
\hline Coef. & -.037792 & $*$ & .1766272 & $* * *$ & .1572088 & $* * *$ & -.0647115 & $*$ & .1909477 & $* *$ & -.4186104 & $* * *$ & -.8203779 & $* * *$ & -.2684125 & $* * *$ \\
\hline Std. Err. & .0624144 & & .0487882 & & .0392953 & & .1460178 & & .0762096 & & .0514676 & & .0493137 & & .0582788 & \\
\hline ESCOLARIDAD & & $\mathbf{P}>\mathbf{Z}$ & & $\mathbf{P}>\mathbf{z}$ & & $\mathbf{P}>\mathbf{Z}$ & & $\mathbf{P}>\mathbf{Z}$ & & $\mathbf{P}>\mathbf{Z}$ & & $\mathbf{P}>\mathbf{Z}$ & & $\mathbf{P}>\mathbf{Z}$ & & $\mathbf{P}>\mathbf{Z}$ \\
\hline \multicolumn{17}{|l|}{ Primaria } \\
\hline Coef. & .0829166 & $*$ & .061736 & $*$ & -.0063284 & $*$ & -.4113422 & $*$ & -.1412191 & $*$ & -.1478463 & $*$ & -.2094731 & $*$ & -.0163479 & $*$ \\
\hline Std. Err. & .2051348 & & .1182939 & & .0827721 & & .2407814 & & .148177 & & .1234672 & & .1226728 & & .0864002 & \\
\hline \multicolumn{17}{|l|}{ Educación No formal } \\
\hline Coef. & 1.227 .812 & $* * *$ & -.2178436 & $*$ & .1719552 & $*$ & -1.227 .471 & $*$ & .087444 & $*$ & -.0045698 & $*$ & -.1116129 & $*$ & -.3465145 & $*$ \\
\hline Std. Err. & .3165788 & & .3389493 & & .2198886 & & 1.037 .797 & & .3612641 & & .3108805 & & .2949757 & & .3935688 & \\
\hline \multicolumn{17}{|l|}{ Bachillerato } \\
\hline Coef. & .395143 & $* *$ & .1710892 & $*$ & .0118806 & $*$ & -1.161 .887 & $* * *$ & -.3067359 & $* *$ & .0188519 & $*$ & -.0012761 & $*$ & -.1840165 & $*$ \\
\hline Std. Err. & .198331 & & .117278 & & .0832771 & & .2501772 & & .151323 & & .124816 & & .1198008 & & .1004479 & \\
\hline \multicolumn{17}{|l|}{ Universitario } \\
\hline Coef. & .9253403 & $* * *$ & .5435671 & $* * *$ & .3324619 & $* * *$ & -.5019994 & $*$ & .0778505 & $*$ & .9785879 & $* * *$ & .3748768 & $* *$ & -.6825856 & $* * *$ \\
\hline Std. Err. & .2032977 & & .1243624 & & .0903411 & & .2755686 & & .1615626 & & .1284558 & & .1257744 & & .1287048 & \\
\hline
\end{tabular}

Nota: * No significativo

** $\quad$ Entre $95 \%$ y $99 \%$

$* * *>$ a $99 \%$ 


\section{UNiversidad SANTOTomas

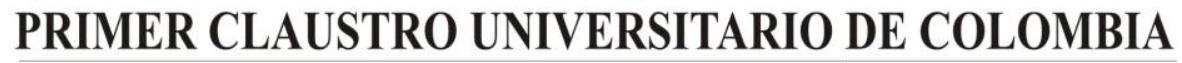 UNIDAD DE INVESTIGACIÓN}

Tabla 6 Ocupación del Área Metropolitana

MODO de TRANSPORTE

\begin{tabular}{|c|c|c|c|c|c|c|c|c|c|c|c|c|c|c|c|c|}
\hline \multirow[b]{2}{*}{ OCUPACION } & \multicolumn{2}{|c|}{ Metro } & \multicolumn{2}{|l|}{ SIT } & \multicolumn{2}{|l|}{ Bus } & \multicolumn{2}{|l|}{ Cable } & \multicolumn{2}{|l|}{ Taxi } & \multicolumn{2}{|c|}{$\begin{array}{c}\text { Auto } \\
\text { Particular }\end{array}$} & \multicolumn{2}{|l|}{ Moto } & \multicolumn{2}{|l|}{ A pie } \\
\hline & & $\mathbf{P}>\mathbf{Z}$ & & $\mathbf{P}>\mathbf{Z}$ & & $\mathbf{P}>\mathbf{Z}$ & & $\mathbf{P}>\mathbf{z}$ & & $\mathbf{P}>\mathbf{Z}$ & & $\mathbf{P}>\mathbf{z}$ & & $\mathbf{P}>\mathbf{Z}$ & & $\mathbf{P}>\mathbf{Z}$ \\
\hline \multicolumn{17}{|l|}{ Desempleado } \\
\hline Coef. & -.5241056 & $*$ & -.6426236 & $* *$ & -.5658648 & $* * *$ & -.148982 & $*$ & -1.277 .175 & $* * *$ & -.5517482 & $* *$ & -.6218919 & $* *$ & -.108517 & $*$ \\
\hline Std. Err. & .4081748 & & .2006245 & & .1466034 & & .5692606 & & .2923843 & & .239337 & & .2192882 & & .2708675 & \\
\hline \multicolumn{17}{|l|}{ Ama de casa } \\
\hline Coef. & -.2069116 & $*$ & -.8017149 & $* * *$ & -.6827412 & $* * *$ & -.2242139 & $*$ & -.5696765 & $* *$ & -.2480675 & $*$ & -.5523111 & $* *$ & -.3711732 & $*$ \\
\hline Std. Err. & .3637273 & & .1776876 & & .1285253 & & .5112805 & & .2059001 & & .1983534 & & .204154 & & .2216251 & \\
\hline \multicolumn{17}{|l|}{ Estudiante } \\
\hline Coef. & .6531682 & $*$ & -.2345012 & $*$ & -.1367913 & $*$ & .6228085 & $*$ & -.8553225 & $* * *$ & .0524787 & $*$ & -.1325056 & $*$ & .9786564 & $* * *$ \\
\hline Std. Err. & .3548962 & & .1692732 & & .1227093 & & .4888566 & & .2037576 & & .1778904 & & .1800665 & & .1586555 & \\
\hline \multicolumn{17}{|l|}{ Trabajador } \\
\hline Coef. & 1.167 .028 & $* *$ & .3482638 & $* *$ & .2059692 & $*$ & .500072 & $*$ & -.2808683 & $*$ & .8025491 & $* * *$ & .8247253 & $* * *$ & .9426226 & $* * *$ \\
\hline Std. Err. & .3502812 & & .1668624 & & .1218797 & & .4825825 & & .1973976 & & .1832275 & & .1809818 & & .1808077 & \\
\hline \multicolumn{17}{|l|}{ Jubilado } \\
\hline Coef. & .0960554 & $*$ & -.449393 & $* *$ & -.5087803 & $* * *$ & -.566531 & $*$ & -.2483132 & $*$ & .0194006 & $*$ & -.4054935 & $*$ & -.365401 & $*$ \\
\hline Std. Err. & .3758354 & & .1938905 & & .1393314 & & .6303008 & & .2131388 & & .2004908 & & .2441342 & & .2625345 & \\
\hline
\end{tabular}

Nota: * No significativo

** Entre $95 \%$ y $99 \%$

$* * *>$ a $99 \%$ 


\section{Universidad SantoTomas

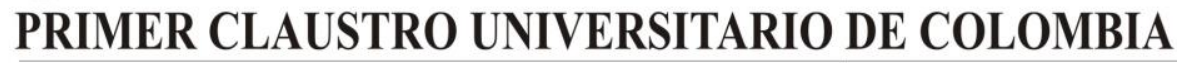 UNIDAD DE INVESTIGACIÓN}

Tabla 7 Tenencia de Vivienda y Constante del modelo del Área Metropolitana

MODO de TRANSPORTE

\begin{tabular}{|c|c|c|c|c|c|c|c|c|c|c|c|c|c|c|c|c|}
\hline \multirow{2}{*}{$\begin{array}{l}\text { TENENCIA } \\
\text { VIVIENDA }\end{array}$} & \multicolumn{2}{|c|}{ Metro } & \multicolumn{2}{|l|}{ SIT } & \multicolumn{2}{|l|}{ Bus } & \multicolumn{2}{|l|}{ Cable } & \multicolumn{2}{|l|}{ Taxi } & \multicolumn{2}{|c|}{$\begin{array}{c}\text { Auto } \\
\text { Particular }\end{array}$} & \multicolumn{2}{|l|}{ Moto } & \multicolumn{2}{|l|}{ A pie } \\
\hline & & $\mathbf{P}>\mathbf{Z}$ & & $\mathbf{P}>\mathbf{Z}$ & & $\mathbf{P}>\mathbf{Z}$ & & $\mathbf{P}>\mathbf{Z}$ & & $\mathbf{P}>\mathbf{z}$ & & $\mathbf{P}>\mathbf{Z}$ & & $\mathbf{P}>\mathbf{Z}$ & & $\mathbf{P}>\mathbf{z}$ \\
\hline otra & & & & & & & & & & & & & & & & \\
\hline Coef. & .0171063 & $*$ & -.147582 & $* *$ & -.0652428 & $*$ & -.4371705 & $* *$ & -.0539357 & $*$ & -.5988176 & $* * *$ & -.1877323 & $* * *$ & -.2117373 & $* * *$ \\
\hline Std. Err. & .0599832 & & .0466186 & & .036859 & & .1416089 & & .0712903 & & .0519857 & & .0448905 & & .0569927 & \\
\hline CONSTANTE & & $\mathbf{P}>\mathbf{z}$ & & $\mathbf{P}>\mathbf{Z}$ & & $\mathbf{P}>\mathbf{Z}$ & & $\mathbf{P}>\mathbf{Z}$ & & $\mathbf{P}>\mathbf{z}$ & & $\mathbf{P}>\mathbf{Z}$ & & $\mathbf{P}>\mathbf{Z}$ & & $\mathbf{P}>\mathbf{Z}$ \\
\hline Coef. & -6.214 .252 & $* * *$ & -334.335 & *** & -2.526 .007 & $* * *$ & -5.653 .124 & $* * *$ & -3.575 .558 & $* * *$ & -3.199 .691 & $* * *$ & -2.609 .752 & $* * *$ & -2.557 .337 & $* * *$ \\
\hline Std. Err. & .4423142 & & .1919621 & & .1362445 & & .6364505 & & .228003 & & .1964485 & & .1842901 & & .173612 & \\
\hline
\end{tabular}

$$
\begin{aligned}
& \text { Nota: * No significativo } \\
& \text { ** } \quad \text { Entre } 95 \% \text { y } 99 \% \\
& * * *>\text { a } 99 \%
\end{aligned}
$$




\section{UNiversidad SANTOTomas

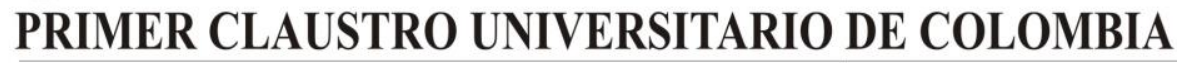 UNIDAD DE INVESTIGACIÓN}

Tabla 8 Rango de Edad de comunidades cercanas a estaciones y usuarios del modo Cable

MODO de TRANSPORTE

\begin{tabular}{|c|c|c|c|c|c|c|c|c|c|c|c|c|c|c|c|c|}
\hline \multirow[b]{2}{*}{ RANGO_EDAD } & \multicolumn{2}{|c|}{ Metro } & \multicolumn{2}{|l|}{ SIT } & \multicolumn{2}{|l|}{ Bus } & \multicolumn{2}{|l|}{ Cable } & \multicolumn{2}{|l|}{ Taxi } & \multicolumn{2}{|c|}{ Auto Particular } & \multicolumn{2}{|l|}{ Moto } & \multicolumn{2}{|l|}{ A pie } \\
\hline & & $\mathbf{P}>\mathbf{Z}$ & & $\mathbf{P}>\mathbf{Z}$ & & $\mathbf{P}>\mathbf{Z}$ & & $\mathbf{P}>\mathbf{Z}$ & & $\mathbf{P}>\mathbf{Z}$ & & $\mathbf{P}>\mathbf{Z}$ & & $\mathbf{P}>\mathbf{Z}$ & & $\mathbf{P}>\mathbf{Z}$ \\
\hline \multicolumn{17}{|l|}{ Entre 15 y 24 años } \\
\hline Coef. & 2.910 .581 & $* *$ & 1.923 .912 & $* * *$ & 1.990 .548 & $* * *$ & 2.782 .839 & $* * *$ & 134.572 & $* *$ & -.3285398 & $*$ & .6268555 & $*$ & -.7723085 & $*$ \\
\hline Std. Err. & 1.068 .882 & & .5408327 & & .3278021 & & .5347159 & & .651437 & & .6187401 & & .3785894 & & .5858154 & \\
\hline \multicolumn{17}{|l|}{ Entre 25 y 34 años } \\
\hline Coef. & 3.460 .943 & $* *$ & 254.686 & $* * *$ & 2.359 .128 & $* * *$ & 2.343 .139 & $* * *$ & 1.968 .924 & $* *$ & .143003 & $*$ & 1.055 .863 & $* *$ & -.1128277 & $*$ \\
\hline Std. Err. & 1.105 .512 & & .5978705 & & .3868508 & & .5835019 & & .7821231 & & .7530916 & & .4375789 & & .794303 & \\
\hline \multicolumn{17}{|l|}{ Entre 35 y 44 años } \\
\hline Coef. & 3.069 .319 & $* *$ & 2.220 .155 & $* * *$ & 2.478 .976 & $* * *$ & 2.436 .529 & $* * *$ & 1.733 .877 & $* *$ & .8661788 & $*$ & .6229146 & $*$ & -.5008224 & $*$ \\
\hline Std. Err. & 1.116 .071 & & .6123126 & & .3935855 & & .5839547 & & .817357 & & .7397977 & & .449838 & & .8534931 & \\
\hline \multicolumn{17}{|l|}{ Entre 45 y 54 años } \\
\hline Coef. & 3.128 .307 & $* *$ & 2.098 .018 & *** & 2.421 .153 & $* * *$ & 2.011 .187 & $* *$ & 1.528 .313 & $*$ & .4015504 & $*$ & .2337977 & $*$ & -.4097054 & $*$ \\
\hline Std. Err. & 1.113 .828 & & .6135361 & & .3912599 & & .5868684 & & .8124699 & & .7481823 & & .4538028 & & .8239113 & \\
\hline \multicolumn{17}{|l|}{ Mayor de 55 años } \\
\hline Coef. & 2.741 .796 & $* *$ & 2.144 .901 & $* * *$ & 2.362 .392 & $* * *$ & 1.707 .905 & $* *$ & 1.727 .998 & $* *$ & -.3575333 & $*$ & -.8794949 & $*$ & -.6736275 & $*$ \\
\hline Std. Err. & 1.116 .592 & & .6028585 & & .3777758 & & .5792632 & & .7651783 & & .7698528 & & .500116 & & .8086532 & \\
\hline
\end{tabular}

Nota: * No significativo

** Entre $95 \%$ y $99 \%$

*** > a $99 \%$ 


\section{Universidad SantoTomas PRIMER CLAUSTRO UNIVERSITARIO DE COLOMBIA UNIDAD DE INVESTIGACIÓN}

Tabla 9 Estrato y Genero de comunidades cercanas a estaciones y usuarios del modo Cable

MODO de TRANSPORTE

\begin{tabular}{|c|c|c|c|c|c|c|c|c|c|c|c|c|c|c|c|c|}
\hline & \multicolumn{2}{|c|}{ Metro } & \multicolumn{2}{|l|}{ SIT } & \multicolumn{2}{|l|}{ Bus } & \multicolumn{2}{|l|}{ Cable } & \multicolumn{2}{|l|}{ Taxi } & \multicolumn{2}{|c|}{ Auto Particular } & \multicolumn{2}{|l|}{ Moto } & \multicolumn{2}{|l|}{ A pie } \\
\hline ESTRATO & & $\mathbf{P}>\mathbf{z}$ & & $\mathbf{P}>\mathbf{Z}$ & & $\mathbf{P}>\mathbf{Z}$ & & $\mathbf{P}>\mathbf{Z}$ & & $\mathbf{P}>\mathbf{z}$ & & $\mathbf{P}>\mathbf{Z}$ & & $\mathbf{P}>\mathbf{Z}$ & & $\mathbf{P}>\mathbf{Z}$ \\
\hline \multicolumn{17}{|l|}{ ESTRATO 2} \\
\hline Coef. & .8159736 & $* *$ & -.030144 & $*$ & -.3882707 & $* *$ & -.3303248 & $*$ & 1.042 .988 & $* *$ & .3128793 & $*$ & .1717452 & * & .8989032 & $* *$ \\
\hline Std. Err. & .2996554 & & .2043804 & & .1442526 & & .1757912 & & .4443159 & & .3401936 & & .1807872 & & .3941297 & \\
\hline \multicolumn{17}{|l|}{ ESTRATO 3} \\
\hline Coef. & 1.226 .929 & *** & .021135 & $*$ & .0947846 & $*$ & -.1471751 & $*$ & 1.995 .644 & $* * *$ & 1.700 .887 & $* * *$ & .5947785 & $* *$ & 2.014 .406 & $* * *$ \\
\hline Std. Err. & .4191765 & & .3530093 & & .2506878 & & .3527204 & & .5352061 & & .4301812 & & .3018347 & & .4923777 & \\
\hline \multicolumn{17}{|l|}{ ESTRATO 4} \\
\hline Coef. & .2669779 & $*$ & -1.872 .366 & $* *$ & -1.139 .109 & ** & -2.414 .259 & $* *$ & 14.375 & ** & .8016282 & $*$ & -.6595475 & $*$ & .52055 & $*$ \\
\hline Std. Err. & .6479321 & & .787183 & & .433148 & & 1.053 .273 & & .7167985 & & .6294892 & & .5213258 & & 1.116 .173 & \\
\hline & & & & & & & & & & & & & & & & \\
\hline \multicolumn{17}{|l|}{ GENERO } \\
\hline \multicolumn{17}{|l|}{ Mujer } \\
\hline Coef. & -.1076681 & $*$ & -.375902 & $* *$ & .02987 & $*$ & -.3622503 & $* *$ & .1549652 & $*$ & -1.031 .099 & $* * *$ & -1.144 .062 & $* * *$ & -.3769105 & $*$ \\
\hline Std. Err. & .2148764 & & .1831744 & & .1371208 & & .1735964 & & .2953935 & & .2809755 & & .168153 & & .2729076 & \\
\hline
\end{tabular}

\begin{tabular}{|c|c|c|}
\hline \multirow[t]{3}{*}{ Nota: } & $*$ & No significativo \\
\hline & $* *$ & Entre $95 \%$ y $99 \%$ \\
\hline & $* * *$ & $>$ a $99 \%$ \\
\hline
\end{tabular}




\section{UnIVERSIDAD SANTOTOMAS

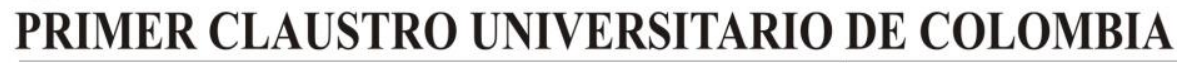 UNIDAD DE INVESTIGACIÓN}

Tabla 10 Escolaridad de comunidades cercanas a estaciones y usuarios del modo Cable

MODO de TRANSPORTE

\begin{tabular}{|c|c|c|c|c|c|c|c|c|c|c|c|c|c|c|c|c|}
\hline \multirow[b]{2}{*}{ ESCOLARIDAD } & \multicolumn{2}{|c|}{ Metro } & \multicolumn{2}{|l|}{ SIT } & \multicolumn{2}{|l|}{ Bus } & \multicolumn{2}{|c|}{ Cable } & \multicolumn{2}{|l|}{ Taxi } & \multicolumn{2}{|c|}{ Auto Particular } & \multicolumn{2}{|l|}{ Moto } & \multicolumn{2}{|l|}{ A pie } \\
\hline & & $\mathbf{P}>\mathbf{Z}$ & & $\mathbf{P}>\mathbf{Z}$ & & $\mathbf{P}>\mathbf{Z}$ & & $\mathbf{P}>\mathbf{Z}$ & & $\mathbf{P}>\mathbf{Z}$ & & $\mathbf{P}>\mathbf{Z}$ & & $\mathbf{P}>\mathbf{Z}$ & & $\mathbf{P}>\mathbf{Z}$ \\
\hline \multicolumn{17}{|l|}{ Primaria } \\
\hline Coef. & .6023314 & $*$ & .1743796 & $*$ & -.0586786 & $*$ & .1244969 & $*$ & -.4336919 & $*$ & -.338189 & $*$ & -.6477808 & $*$ & -.4599667 & $*$ \\
\hline Std. Err. & .7688009 & & .449084 & & .2560292 & & .3519643 & & .4374836 & & .4795323 & & .3362862 & & .367812 & \\
\hline \multicolumn{17}{|l|}{ Educación No formal } \\
\hline Coef. & 2.595 .929 & $* *$ & 1.273 .751 & $*$ & .1590711 & $*$ & -.274152 & $*$ & -1.427 .871 & $*$ & -1.381 .761 & $*$ & 1.389 .461 & $*$ & -1.387 .467 & $*$ \\
\hline Std. Err. & 1.069 .507 & & .9694454 & & .7961037 & & 1.169 .333 & & 1.307 .886 & & 1.420 .486 & & .7872409 & & 1.740 .179 & \\
\hline \multicolumn{17}{|l|}{ Bachillerato } \\
\hline Coef. & 111.826 & $*$ & .4648006 & $*$ & .1479547 & $*$ & -.1148282 & $*$ & -.4419376 & $*$ & .0602483 & $*$ & -.0867202 & * & -.5008832 & * \\
\hline Std. Err. & .7643194 & & .4555858 & & .2683189 & & .3657165 & & .472406 & & .5040313 & & .3406858 & & .4614633 & \\
\hline \multicolumn{17}{|l|}{ Universitario } \\
\hline Coef. & 1.504 .664 & $*$ & 1.569 .143 & ** & .8749645 & $* *$ & .0205748 & $*$ & -.1396802 & $*$ & .8204228 & $*$ & .7054377 & $*$ & -.4166043 & $*$ \\
\hline Std. Err. & .793653 & & .4836778 & & .3078631 & & .4223316 & & .5635846 & & .5591415 & & .3787829 & & .6283599 & \\
\hline
\end{tabular}

Nota: * No significativo

** $\quad$ Entre $95 \%$ y $99 \%$

$* * *>$ a $99 \%$ 


\section{Wiveriversidad SANTOTomas

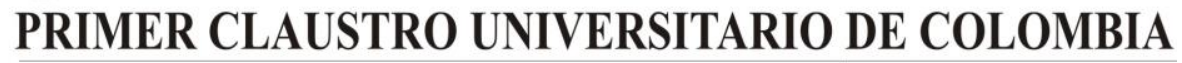 UNIDAD DE INVESTIGACIÓN}

Tabla 11 Ocupación de comunidades cercanas a estaciones y usuarios del modo Cable

MODO de TRANSPORTE

\begin{tabular}{|c|c|c|c|c|c|c|c|c|c|c|c|c|c|c|c|c|}
\hline \multirow[b]{2}{*}{ OCUPACION } & \multicolumn{2}{|c|}{ Metro } & \multicolumn{2}{|l|}{ SIT } & \multicolumn{2}{|l|}{ Bus } & \multicolumn{2}{|c|}{ Cable } & \multicolumn{2}{|l|}{ Taxi } & \multicolumn{2}{|c|}{ Auto Particular } & \multicolumn{2}{|l|}{ Moto } & \multicolumn{2}{|l|}{ A pie } \\
\hline & & $\mathbf{P}>\mathrm{Z}$ & & $\mathbf{P}>\mathbf{Z}$ & & $\mathbf{P}>\mathbf{Z}$ & & $\mathbf{P}>\mathbf{Z}$ & & $\mathbf{P}>\mathbf{Z}$ & & $\mathbf{P}>\mathbf{Z}$ & & $\mathbf{P}>\mathbf{z}$ & & $\mathbf{P}>\mathbf{z}$ \\
\hline \multicolumn{17}{|l|}{ Desempleado } \\
\hline Coef. & -.0638783 & $*$ & -.7307025 & $*$ & -.2637267 & $*$ & -.2689365 & $*$ & -.0247167 & $*$ & -.6075395 & $*$ & .7020811 & $*$ & -.7076346 & $*$ \\
\hline Std. Err. & 1.145 .354 & & .9224596 & & .48058 & & .6146613 & & 1.299 .571 & & 1.087 .644 & & .9026785 & & 1.253 .006 & \\
\hline \multicolumn{17}{|l|}{ Ama de casa } \\
\hline Coef. & -.2457472 & $*$ & -.4368208 & $*$ & -.6747243 & $*$ & -.4065358 & $*$ & .9599754 & $*$ & .3059584 & $*$ & 1.124 .782 & $*$ & -1.022 .229 & $*$ \\
\hline Std. Err. & 1.093 .588 & & .8253365 & & .4492745 & & .583686 & & 1.128 .719 & & .9200661 & & .8674354 & & 107.855 & \\
\hline \multicolumn{17}{|l|}{ Estudiante } \\
\hline Coef. & .0321957 & $*$ & .2295293 & $*$ & -.0288525 & $*$ & -.8727004 & $*$ & .7575718 & $*$ & -.2631866 & $*$ & .9280473 & $*$ & .2693373 & $*$ \\
\hline Std. Err. & 1.077 .163 & & .7813291 & & .4238199 & & .5498306 & & 1.081 .023 & & .7009337 & & .7608463 & & .5331873 & \\
\hline \multicolumn{17}{|l|}{ Trabajador } \\
\hline Coef. & 1.540 .141 & $*$ & 1.593 .422 & $* *$ & 1.123 .666 & $* *$ & 1.377 .883 & $* *$ & 1.474 .837 & $*$ & 1.239 .837 & $*$ & 325.361 & $* * *$ & 1.270 .997 & $*$ \\
\hline Std. Err. & 1.057 .164 & & .7739404 & & .4255866 & & .5437276 & & 111.128 & & .8333797 & & .8000593 & & .8023159 & \\
\hline \multicolumn{17}{|l|}{ Jubilado } \\
\hline Coef. & -.3140515 & $*$ & .39371 & $*$ & .0622828 & $*$ & -.0173875 & $*$ & 1.521 .218 & $*$ & .7250773 & $*$ & .280962 & $*$ & -.4271873 & $*$ \\
\hline Std. Err. & 1.287 .335 & & .8795636 & & .5017394 & & .7051871 & & 1.171 .163 & & 1.012 .705 & & 1.308 .448 & & 132.647 & \\
\hline
\end{tabular}

Nota: * No significativo

** Entre $95 \%$ y $99 \%$

$* * *>$ a $99 \%$ 


\section{Universidad SantoTomas PRIMER CLAUSTRO UNIVERSITARIO DE COLOMBIA UNIDAD DE INVESTIGACIÓN}

Tabla 12 Tenencia de Vivienda y Constante del modelo de comunidades cercanas a estaciones y usuarios del modo Cable

MODO de TRANSPORTE

\begin{tabular}{|c|c|c|c|c|c|c|c|c|c|c|c|c|c|c|c|c|}
\hline & \multicolumn{2}{|l|}{ Metro } & \multicolumn{2}{|l|}{ SIT } & \multicolumn{2}{|l|}{ Bus } & \multicolumn{2}{|l|}{ Cable } & \multicolumn{2}{|l|}{ Taxi } & \multicolumn{2}{|c|}{ Auto Particular } & \multicolumn{2}{|l|}{ Moto } & \multicolumn{2}{|c|}{ A pie } \\
\hline VIVIENDA & & $\mathbf{P}>\mathbf{Z}$ & & $\mathbf{P}>\mathbf{Z}$ & & $\mathbf{P}>\mathbf{Z}$ & & $\mathbf{P}>\mathbf{Z}$ & & $\mathbf{P}>\mathbf{Z}$ & & $\mathbf{P}>\mathbf{Z}$ & & $\mathbf{P}>\mathbf{Z}$ & & $\mathbf{P}>\mathbf{Z}$ \\
\hline otra & & & & & & & & & & & & & & & & \\
\hline Coef. & -.0146689 & $*$ & .1650969 & $*$ & .599998 & $* * *$ & .5118368 & $* *$ & .6979054 & $* *$ & .2170625 & $*$ & .440295 & $* *$ & .1465039 & $*$ \\
\hline Std. Err. & .2038634 & & .1754652 & & .1337052 & & .1665052 & & .288896 & & .2494385 & & .1529583 & & .2653755 & \\
\hline CONSTANTE & & & & & & & & & & & & & & & & \\
\hline Coef. & -7.068 .929 & $* * *$ & -4.613 .482 & $* * *$ & \begin{tabular}{|l|}
-3.312 .954 \\
\end{tabular} & $* * *$ & -3.634 .045 & $* * *$ & -6.212 .888 & $* * *$ & -3.224 .028 & $* * *$ & -3.470 .594 & $* * *$ & -320.916 & $* * *$ \\
\hline Std. Err. & 1.495 .543 & & .8709128 & & \begin{tabular}{|c|}
4729204 \\
\end{tabular} & & .6428541 & & 1.162 .429 & & .6915391 & & .7458506 & & .6173471 & \\
\hline
\end{tabular}

\begin{tabular}{|c|c|c|}
\hline \multirow[t]{3}{*}{ Nota: } & $*$ & No significativo \\
\hline & $* *$ & Entre $95 \%$ y $99 \%$ \\
\hline & $* * *$ & $>$ a $99 \%$ \\
\hline
\end{tabular}




\section{Universidad SANTOTOMas PRIMER CLAUSTRO UNIVERSITARIO DE COLOMBIA UNIDAD DE INVESTIGACIÓN}

\subsection{Interpretación del modelo y Conclusiones}

\subsection{8 observaciones (Área Metropolitana del Valle de Aburrá)}

Como interpretación de resultados y conclusiones se establece que el rango de edad para la el modo sistema de transporte de las observaciones estimadas, solo es significativo para las personas que tienen un rango de edad de entre 15 y 24 años y para los mayores de 55 años que cuentan con vehículo particular y los propietarios de moto mayores de 55 años. Las personas encuestadas que tienen un rango de edad de entre 25 y 34 años y 45 y 54 años, son las personas que significativamente utilizan el modo de transporte caminar.

En cuanto al estrato socioeconómico, se analiza que para los estratos 2 y 3 el resultado es significativo y tienen más probabilidad de utilización de los sistemas SIT y Bus. El sistema metro tiene mayor probabilidad de escogerse si se tiene en cuenta un aumento en el estrato. La moto tiene a su vez una probabilidad mayor de escogerse a medida que aumenta el estrato.

Las mujeres tienen 3,7\% menor probabilidad de utilizar el metro con respecto a los hombres, un 6,4\% menos de probabilidad de utilización del sistema cable, y es muy interesante conocer que las mujeres tienen una probabilidad del 19\% mas de utilizar el modo taxi que los hombres.

El aumento de la escolaridad es relativamente significativo y especialmente en el sistema metro, se evidencia que un aumento en el nivel de escolaridad, aumenta la probabilidad de utilización del modo de transporte. El poseer estudios universitarios aumenta la probabilidad de contar con un vehículo particular para su desplazamiento en un 97\%, mientras que caminar aumenta la probabilidad entre menor escolaridad se posea.

En cuanto a la ocupación de las observaciones, se concluye que los estudiantes, trabajadores y jubilados tienen una mayor probabilidad de utilizar el sistema metro y que ser estudiante y trabajador aumenta las probabilidades de utilizar el sistema cable. Cuando se es jubilado, se tiene una probabilidad mayor de escoger el vehículo particular.

En cuanto a la propiedad de la vivienda, las personas que utilizan el metro tienen más probabilidad de poseer una vivienda propia, mientras que la utilización de SIT, bus, cable y taxi aumenta la probabilidad de no poseer vivienda propia. 


\section{UNIVERSIDAD SANTO TOMAS PRIMER CLAUSTRO UNIVERSITARIO DE COLOMBIA UNIDAD DE INVESTIGACIÓN}

\subsection{4 observaciones (Barrios aledaños al sistema cable e individuos que utilizan cable)}

Para las observaciones de las personas que habitan en barrios colindantes con las estaciones de cable, se evidencia que son significativos por la edad y que a medida que aumenta existe una probabilidad mayor de utilizar este sistema. La probabilidad de utilización del cable aumenta después de los 45 años. Utilizar taxi, aumenta significativamente la probabilidad a medida que aumenta la edad. El uso de vehículo particular aumenta la probabilidad de utilización para las edades entre 25 y 54 años. La utilización de moto aumenta la probabilidad para los rangos de edad menores a 54 años.

La variable estrato socioeconómico muestra que aumenta la probabilidad de utilización de sistema metro a medida que este aumenta. La probabilidad de utilización del modo taxi, también aumenta con el estrato. La probabilidad de utilizar moto, aumenta en los estratos 2 y 3. Para la utilización del cable tiene una probabilidad menor a medida que el estrato disminuye.

Las mujeres tienen mayor probabilidad de utilizar taxi o bus con respecto a los hombres y menor probabilidad de utilizar el metro, SIT, cable o caminar.

La escolaridad es significativa para la utilización del sistema metro y el SIT. Tener estudios de primaria y universitarios aumenta la probabilidad de utilización del sistema cable. Entre menos escolaridad se posea, existe una menor probabilidad de utilizar el taxi. Las personas que poseen títulos universitarios tienen un $82 \%$ mas de probabilidad de utilizar el vehículo particular como medio de transporte.

Ser estudiante, trabajador o jubilado, aumenta la probabilidad de utilizar el metro. En el sistema cable, ser trabajador aumenta la probabilidad de utilización de este modo de transporte. Utilizar la moto no es significativo para los trabajadores, pero aumenta la probabilidad de utilización entre los desempleados, estudiantes, amas de casa y jubilados. Caminar es significativo para los estudiantes y trabajadores.

La propiedad de la vivienda es en la mayoría de modos de transporte significativa, las personas que utilizan metro tienen mayor probabilidad de tener vivienda propia. Mientras que, quienes utilizan SIT, cable, taxi, auto particular, moto y caminar, tienen una probabilidad mayor de no poseer vivienda propia. 


\section{Uiversidad Santo Tomas PRIMER CLAUSTRO UNIVERSITARIO DE COLOMBIA UNIDAD DE INVESTIGACIÓN}

\subsubsection{Conclusiones de la observación del Área Metropolitana con respecto a las viviendas y usuarios del sistema cable.}

La utilización del sistema cable para los rangos de edad, no son significativos en las observaciones generales con respecto a los barrios colindantes. No existe evidencia empírica de una relación del comportamiento del Área Metropolitana con relación a barrios colindantes de sistema cable

Puesto que el sistema cable es implementado en comunidades con menores condiciones socioeconómicas, se evidencia que la utilización del sistema es mayoritariamente utilizado por los estratos 2, 3 y 4, de aquí se establece que no existen los estratos 5 y 6 dentro de las comunidades colindantes a los sistemas cables. Se evidencia un comportamiento similar a las observaciones del Área Metropolitana con respecto a los barrios colindantes en cuanto a que existe una mayor probabilidad de utilizar el sistema a medida que disminuye el estrato.

Las mujeres evidencian mayor probabilidad de utilizar taxi en ambas observaciones y también mayor probabilidad de utilizar el bus con respecto a los hombres, así mismo tienen una menor probabilidad de utilizar el metro y el cable.

Se evidencia que, a mayor escolaridad, existe una mayor probabilidad de utilizar el metro y el comportamiento muestra la misma tendencia de aumentar la probabilidad de contar con vehículo particular si se cuenta con estudios universitarios.

La ocupación presenta el mismo comportamiento, que como se evidencia en los resultados, quienes utilizan el sistema metro tienen mas probabilidad si son estudiantes, trabajadores o jubilados. Se evidencia un comportamiento distinto para la utilización de la moto, puesto que en los barrios aledaños a los sistemas cables, la probabilidad de utilizar moto aumenta con relación a la ocupación, mientras que en el Área Metropolitana disminuye esta opción.

Ser propietario de una vivienda en barrios aledaños, aumenta la probabilidad de utilización del sistema cable, mientras que para las observaciones de toda el Área Metropolitana esta probabilidad disminuye.

En concordancia con lo anterior se puede estimar que la elección del modo de transporte es significativa para quienes poseen vivienda propia en el sistema cable y que habitan en barrios colindantes a los sistemas cables, adicional a ello concluir que la ocupación es significativa y presenta mayor probabilidad de utilización del sistema metro.

Se recomienda realizar unas encuestas objetivo para determinar la tenencia de la vivienda en los hogares establecidos a menos de $1 \mathrm{~km}$ de distancia de las estaciones del sistema cable, así 


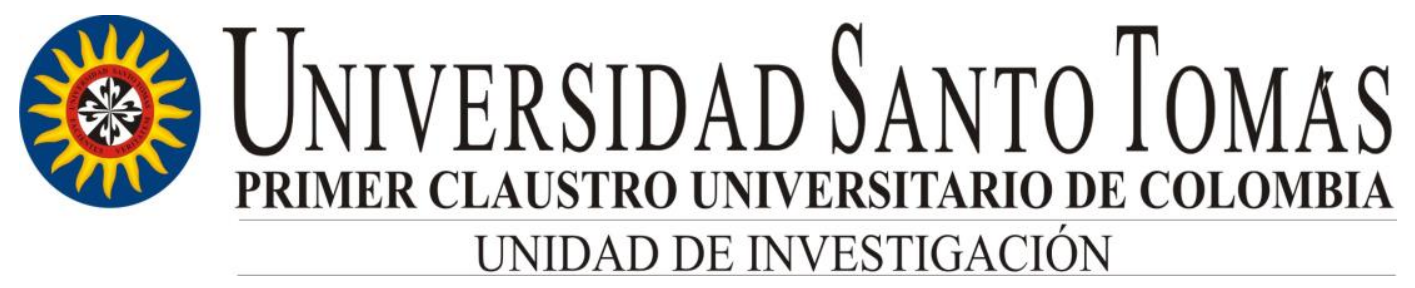

como la ocupación, escolaridad y rango de edad; de este modo se puede estimar si la calidad de vida de la comunidad mejora con la implementación de estos sistemas.

Por otra parte, se recomienda realizar un inventario de inversión económica privada, en una distancia no mayor a 100 metros de las estaciones de los sistemas: metro, cable, tranvía que podrán estimar si efectivamente la inversión privada aumenta, tal como lo demuestra la evidencia empírica y el estudio de Leinberger (2014). 


\section{Bibliografía}

(13 de Septiembre de 2019). Obtenido de Área Metropolitana Valle de Aburrá: https://www.metropol.gov.co/movilidad/Paginas/transporte-publico/sitva.aspx

Acemoglu, D., \& Robinson, J. (2012). Por qué fracasan los países: Los orígenes del poder, la prosperidad y la pobreza. (M. García, Trad.) Barcelona: Deusto S.A. Ediciones.

Agostini, C., \& Palmucci, G. (2008). Capitalización anticipada del metro de santiago en el precio de las viviendas. El Trimestre Económico, 75(298), 403-431.

Arango Z., C. (1981). Crónicas de la lucha por la vivienda en Colombia (1a ed.). Bogotá: Centro Nacional Provivienda.

Ascher, F. (2005). Ciudades con velocidad y movilidad múltiples:. ARQ (Santiago)(60), 1119.

Boarnet, M. (1994). The Monocentric Model and Employment Location. Journal of Urban Economics, 36, 79-97.

Boarnet, M., \& Geho, E. (2005). Specification Issues in Models of Population and Employment Growth. Papers in Regional Science(84), 21-46.

Carlino, G., \& Mills, E. (1987). The Determinants of County Growth. Journal of Regional Science, 27, 39-54.

Condom, P., Gruenberger, S., \& Klaptocz, M. (May de 2008). The Case for the Tram: Learning from Portland. SUSTAINABILITY BY DESIGN(6).

Cortés, A., \& Figueroa , C. (2012). Encadenamiento de desplazamientos: el transporte público de santiago como productor de actividad comercial. Revista Transporte y Territorio(7), 166-183.

Currie, G., \& Stanley, J. (2008). Investigating Links between Social Capital and Public Transport. Journal Transport Reviews, 28, 529-547.

Davies, L., Banister, D., \& Hall, P. (2004). Transport and City Competitiveness - Literature Review . School of Planning, University College, .

De Grange, L. (2010). El gran impacto del Metro. EURE Revista Latinoamericana de Estudios Urbano Regionales, 36(107). 
Departamento Administrativo Nacional de Estadistica DANE. (s.f.). www.dane.gov.co. Obtenido de http://www.dane.gov.co/index.php/estadisticas-por-tema/demografia-ypoblacion/proyecciones-de-poblacion

Estupiñan, N. (Enero-Junio de 2011). Impactos en el uso del suelo por inversiones de transporte público masivo. Revista de ingeniería, Universidad de los Andes(33), 3443.

Fajardo Hoyos, C., \& Gómez Sánchez, A. (2015). Análisis de la elección modal de transporte público y privado en la ciudad de Popayán. Territorios, 33, 157-190.

Fisher, P., \& Peters, A. (1998). Industrial Incentives: Competition Among American States and Cities. Kalamazoo, Michigan: W.E. Upjohn Institute for Employment.

Flamm, M., Jemelin, C., \& Kaufmann, V. (2007). Combining person based GPS tracking and prompted recall interviews for a comprehensive investigation of travel behaviour adaptation processes during life course transitions. Swiss Transport Research Conference. Monte Verità / Ascona.

Greene, W. (1998). Análisis Econométrico. Madrid: Prentice Hall Inc.

Greene, W. (2012). Econometric Analysis. Upper Saddle River, NJ: Prentice Hall.

Hensher, D. (1986). Sequential and Full Information Maximum Likelihood Estimation of a Nested Logit Mode. The Review of Economics and Statistics, 68(4), 657-667.

Hensher, D. (1986). Sequential and Full Information Maximum Likelihood Estimation of a Nested Logit Model. The Review of Economics and Statistics, 68(4), 657-667.

Hensher, D. (1986). Sequential and Full Information Maximum Likelihood Estimation of a Nested Logit Model. The Review of Economics and Statistics, 68(4), 657-667.

Hernández, D. (2012). Activos y estructuras de oportunidades de movilidad. Una propuesta analítica para el estudio de la accesibilidad por transporte público, el bienestar y la equidad. EURE, 38(115), 117-135.

Holl, A. (2004). Transport Infrastructure, Agglomeration Economies, and Firm Birth: Empirical Evidence from Portugal. Journal of Regional Science(44), 693-712.

Kaufmann, V., Bergman, M., \& Joye, D. (2004). Motility: mobility as capital. International Journal of Urban and Regional Research, 28(4), 745-756.

Keyes, C. (1998). Social Well-Being. Social Psychology Quarterly, 61(2), 121-140. 
Keyes, C., Shmotkin, D., \& Ryff, C. (2002). Optimizing well-being: the empirical encounter of two traditions. Journal of Personality and Social Psychology, 1007-1022.

Leinberger, C. B. (14 de November de 2014). Riding light rail and streetcars into better communities. Obtenido de https://www.washingtonpost.com/opinions/riding-lightrail-and-streetcars-into-better-communities/2014/11/14/58fd98b2-6a86-11e4-a31c77759fc1eacc_story.html?utm_term=.0730f8ea2061

Maciulis, A., Vasilis, A., \& Jakubauskas, G. (2009). The impact of transport on the competitiveness of national economy. Journal Transport, 24, 93-99.

Martinez, F., De Cristófaro, G., Sánchez, L., Hantke, B., \& Diaz, A. (2014). Análisis de situación, problemáticas y propuestas para el Sistema de Transporte en Argentina. Buenos Aires.

Massot, M., \& Orfeuil, J. (2005). La mobilité au quotidien, entre choix individuel et production sociale. Cahiers internationaux de sociologie(118), 81-100.

McFadden, D. (1984). Econometric analysis of qualitative response models, Handbook of Econometrics (1 ed., Vol. 2). Amsterdam.

Mcguckin, N., \& Murakami, E. (1999). Examining trip-chaining behavior: comparison of travel by men and women. Transportation Research Record, 1693, 79-85.

Metro de Medellin. (s.f.). www.metrodemedellin.gov.co. Obtenido de https://www.metrodemedellin.gov.co/qui\%C3\%A9nessomos/historia

Molina, M., \& Fernández, D. (2013). Propuesta tranvía para Tunja a partir de caracterización de flujos vehiculares y analisis de indicadores de movilidad urbana. Tunja.

Moser, C. (1998). The asset vulnerability framework: Reassessing urban poverty reduction strategies. World Development, 26(1), 1-19.

Mosquera Abadía, H. A. (Enero-Junio de 2015). Dimensiones que conforman el riesgo de exclusión en el sistema de. Cuadernos de Administración, 31(53), 108-116.

Naciones Unidas. (2012). Objetivos de Desarrollo Sostenible. Río de Janeiro. Obtenido de http://www.undp.org/content/undp/es/home/sustainable-development-goals.html

North, D. C. (1993). Instituciones, cambio institucional y desempeño económico. (A. Bárcena, Trad.) México: FCE. 
Partridge, M. D., Rickman, D. S., Ali, K., \& Olfert, R. (2008). The Geographic Diversity of U.S. Nonmetropolitan Growth Dynamics: A Geographically Weighted Regression Approach. Land Economics, 84(2), 241-266.

Rubio, C. (2016). La Gran Pacificación (Taiheiki). Madrid: Trotta.

Santos y Ganges, L., \& de las Rivas Sanz, J. (2008). Ciudades con atributos: conectividad, accesibilidad y movilidad. Ciudad e infraestructuras(11), 13-32.

Schonfelder, S., \& Axhausen, K. (October de 2003). Activity spaces: measures of social exclusion? Transport Policy, 10, 273-286.

Stiglitz, J., Sen, A., \& Fitoussi, J.-P. (2009). Report by the Commission on the Measurement of Economic Performance and Social Progress. Obtenido de http://ec.europa.eu/eurostat/documents/118025/118123/Fitoussi+Commission+repor $\mathrm{t}$

STORE, T. C. (29 de 12 de 2017). Cable Car History. Obtenido de Cable Car Store: https://www.cablecarstore.com/history.html

Urrego, G. A. (2010). Sistemas integrados de transporte masivo en colombia: avances, retos y perspectivas en el marco de la política nacional de transporte urbano . Bogotá: Contraloria General de la Republica.

Urry, J., Shove, E., \& Cass, N. (2005). "Social exclusion, mobility and access". The Sociological Review, 53(3), 539-555.

Vásquez, J. (2008). Microeconometría Aplicada. Santiago de Chile. 